\title{
Minimum action method for the Kardar-Parisi-Zhang equation
}

\author{
Hans C. Fogedby* \\ Department of Physics and Astronomy, \\ University of Aarhus \\ Ny Munkegade, 8000, \\ Aarhus C, Denmark \\ and \\ Niels Bohr Institute \\ Blegdamsvej 17, 2100, \\ Copenhagen Ø, Denmark \\ Weiqing Rent \\ Courant Institute of Mathematical Sciences, New York University \\ 251 Mercer Street, New York, NY 10012, US
}

\begin{abstract}
We apply a numerical minimum action method derived from the Wentzell-Freidlin theory of large deviations to the Kardar-Parisi-Zhang equation for a growing interface. In one dimension we find that the switching scenario is determined by the nucleation and subsequent propagation of facets or steps, corresponding to moving domain walls or growth modes in the underlying noise driven Burgers equation. The transition scenario is in accordance with recent analytical studies of the one dimensional Kardar-Parisi-Zhang equation in the asymptotic weak noise limit. We also briefly discuss transitions in two dimensions.
\end{abstract}

PACS numbers: 05.40.-a, 02.50.-r, 05.45.Yv, 05.90.+m

*Electronic address: fogedby@phys.au.dk

$\dagger$ Electronic address: weiqing@cims.nyu.edu 


\section{INTRODUCTION}

The large majority of natural phenomena are characterized by being out of equilibrium. This class includes turbulence in fluids, interface and growth problems, chemical reactions, processes in glasses and amorphous systems, biological processes, and even aspects of economical and sociological structures [1, 2]. In this context there is a continuing interest in the strong coupling aspects of stochastically driven non equilibrium model systems [3, 4]. Here the dynamics of complex systems driven by weak noise, corresponding to rare events, is of particular interest. The issue of different time scales characterizes many interesting processes in nature. For instance, in the case of chemical reactions the reaction time is often orders of magnitude larger than the molecular vibration periods [5]. The time scale separation problem is also encountered in the case of conformational changes of biomolecules [6], nucleation events during phase transitions, switching of the magnetization in magnetic materials [7, 8], and even in the case of comets exhibiting rapid transitions between heliocentric orbits around Jupiter [9].

In the weak noise limit the standard Monte Carlo method or direct simulation of the Langevin equation becomes impractical owing to the large separation of time scales and alternative methods have been developed. The most notable analytical approach is the formulation due to Freidlin and Wentzel which yields the transition probabilities in terms of an action functional [10]. This approach is the analogue of the variational principle proposed by Machlup and Onsager [11, 12], see also work by Graham et al. [13, 14] and Dykman [15]. The Freidlin-Wentzel approach is also equivalent to the Martin-Siggia-Rose method [16] in the weak noise limit of the path integral formulation [17, 18, 19, 20, 21]. In order to overcome the time scale gap various numerical methods have also been proposed. We mention here the transition path sampling method [22] and the string method [23, 24, 25, 26].

A particularly interesting non equilibrium problem of relevance in the nanophysics of magnetic switches is the influence of thermal noise on two-level systems with spatial degrees of freedom [7, 8, 27]. In a recent paper by E, Ren, and Vanden-Eijnden [28], see also Ref. [29], this problem has been addressed using the Ginzburg-Landau (GL) equation driven by thermal noise. Applying the field theoretic version of the Onsager-Machlup functional [11, 12] in the Freidlin-Wentzell formulation [10], these authors developed the so called minimum action method in which they implemented a powerful numerical optimization techniques for 
the determination of the space-time configuration which minimizes the Freidlin-Wentzell action. The minimizers correspond to the kinetic pathways and the associated action yields the switching probabilities in the long time-low temperature limit. In the picture emerging from the numerical study the switching between metastable states is due to noise induced nucleation and subsequent propagation of domain walls across the sample. Subsequently, we supplemented the work by E et al. and presented a dynamical description and analysis of the non equilibrium transitions in the noisy 1D GL equation for an extensive system based on a weak noise canonical phase space formulation of the Freidlin-Wentzel or Martin-SiggiaRose methods [30, 31]. We derived propagating nonlinear domain wall or soliton solutions of the resulting canonical field equations with superimposed diffusive modes. The transition pathways are characterized by the nucleations and subsequent propagation of domain walls. We discussed the general switching scenario in terms of a dilute gas of propagating domain walls and evaluated the Arrhenius factor in terms of the associated action. In conclusion we found excellent agreement with the numerical studies by E et al. [28, 29].

The noise-driven GL equation belongs to the class of so-called gradient systems where the drift term in the Langevin equation can be derived from a free energy functional. Regarding the kinetic transitions this property implies the existence of an underlying free energy landscape in which the optimal pathway proceeds via saddle points, yielding the corresponding Arrhenius factor. We note that this interpretation implies a fluctuation-dissipation theorem relating the strength of the noise to the kinetic transport coefficient. There is, however, another interesting class of stochastic model systems characterized by Langevin equations, where the drift term cannot be associated with a free energy functional. Those are the so called non-gradient systems for which the interpretation of pathways in a free energy landscape fails and has to be replaced by pathways in an "action landscape". In recent work, see e.g. Refs. [32, 33], where earlier references can be found, we have addressed a non equilibrium model falling in the class of non-gradient systems, namely the Kardar-Parisi-Zhang (KPZ) equation or, equivalently, noisy Burgers equation describing, for example, a growing interface. Using the weak noise canonical phase space method alluded to above, we find that the kinetic pathways correspond to nucleation and propagation of localized growth modes with superimposed diffusive modes. The growth modes together with the diffusive modes carry an action, yielding the transition probabilities.

The purpose of the present paper is to attempt to substantiate the weak noise growth 
mode approach to the KPZ equation by a direct numerical optimization employing the minimum action method developed by E, Ren, and Vanden-Eijnden [28], see also Refs. [34, 35]. Similar to the GL case we find in 1D that the switching scenario is determined by the nucleation and propagation of growth modes. We are also able to account numerically for the associated transition probabilities. The paper is organized in the following manner. In Sec. II] we briefly review the KPZ equation and the analytical results obtained by the weak noise canonical phase space approach. In Sec. III we introduce the minimum action method and establish the connection with the phase space method and path integral formulations. In Sec. IV] we discuss the numerical implementation of the Freidlin-Wentzel scheme. In Sec. V] we present the numerical results for various switching scenarios. In Sec. VI we offer a heuristic discussion of the numerical data based on the analytical phase space method. In Sec. VII we briefly discuss kinetic pathways in 2D and present some switching scenarios. Sec. VIII is devoted to a summary and a conclusion.

\section{THE KPZ EQUATION}

In this section we review the KPZ equation and apply the weak noise method. The KPZ equation describes an intrinsic non equilibrium problem and plays in some sense the same role as the Ginzburg-Landau functional in equilibrium physics [2, 36]. The KPZ equation was introduced in 1986 in a seminal paper by Kardar, Parisi, and Zhang [37], see also Ref. [38, 39], and purports to describe non equilibrium aspects of a growing interface [4, 40]. In a Monge representation the KPZ equation for the stochastic time evolution of the height field $h(\mathbf{r}, t)$ has the form

$$
\begin{aligned}
& \frac{\partial h}{\partial t}=\nu \nabla^{2} h+\frac{\lambda}{2}(\nabla h)^{2}-F+\eta \\
& \langle\eta \eta\rangle(\mathbf{r}, t)=\Delta \delta^{d}(\mathbf{r}) \delta(t) .
\end{aligned}
$$

Here the damping coefficient or viscosity $\nu$ characterizes the linear diffusion term $\nu \nabla^{2} h$, the growth parameter $\lambda$ controls the strength of the nonlinear growth term $(\lambda / 2)(\boldsymbol{\nabla} h)^{2}, F$ is the imposed drift term and is a constant here, and $\eta$ a locally correlated white Gaussian noise, modelling the stochastic nature of the drive or environment; the noise correlations are characterized by the noise strength $\Delta$. 


\section{A. Burgers and Cole-Hopf equations}

In the growth mode analysis of the KPZ equation the local slope of the growing interface given by the vector field,

$$
\mathbf{u}=\nabla h
$$

is of importance. In terms of $\mathbf{u}$ the $\mathrm{KPZ}$ equation then maps onto the Burgers equation driven by conserved noise [41, 42, 43, 44]

$$
\frac{\partial \mathbf{u}}{\partial t}=\nu \nabla^{2} \mathbf{u}+\lambda(\mathbf{u} \cdot \nabla) \mathbf{u}+\nabla \eta
$$

In the deterministic case for $\eta=0$ the Burgers equation has been used to study irrotational fluid motion and turbulence [45, 46, 47, 48, 49]; it has also played a role in astrophysics in the context of large scale structures in the universe [50, 51].

Another quantity of importance in our analysis of the KPZ equation is the diffusive field $w$ defined by the non-linear Cole-Hopf transformation [38, 52, 53]

$$
w=\exp \left[\frac{\lambda}{2 \nu} h\right] .
$$

In terms of $w$ the KPZ equation maps onto a linear diffusion equation driven by a multiplicative noise, here denoted the Cole-Hopf $(\mathrm{CH})$ equation,

$$
\frac{\partial w}{\partial t}=\nu \nabla^{2} w-\frac{\lambda}{2 \nu} w F+\frac{\lambda}{2 \nu} w \eta
$$

In the absence of noise for $\eta=0$ the $\mathrm{CH}$ equation reduces to the linear diffusion equation and is readily analyzed permitting a complete discussion of the KPZ and Burgers equations in the deterministic case [37, 38]. In the noisy case a path integral representation maps the $\mathrm{CH}$ equation and consequently the KPZ equation onto a model of a directed polymer in a quenched random potential. The disordered directed polymer constitutes a toy model within the spin glass literature and has been analyzed by means of replica, Bethe ansatz, and functional renormalization group techniques [39, 54, 55, 56].

\section{B. Scaling properties}

Most work on the KPZ equation has addressed the scaling issues. For completeness we summarize the salient features here. The KPZ equation lives at a critical point and conforms 
to the dynamical scaling hypothesis [4, 57, 58, 59] which in terms of the height correlations assumes the form:

$$
\langle h h\rangle(\mathbf{r}, t)=r^{2 \zeta} f\left(t / r^{z}\right) .
$$

Here $\zeta$ is the roughness exponent, $z$ the dynamical exponent, and $f$ the associated scaling function. The exponent $\zeta$ is a measure of the roughness of the interface, e.g., for $\zeta=0$ the interface is flat, for $\zeta=1 / 2$ the interface exhibits a random walk profile, $\langle h h\rangle(\mathbf{r}) \sim r$. The exponent $z$ is a measure of the dynamical scaling, e.g., for diffusive behavior $z$ locks onto 2; for a $1 \mathrm{D}$ growing interface $z=3 / 2$.

In order to extract scaling properties the initial analysis of the KPZ equation was based on the dynamic renormalization group (DRG) method, previously developed and applied to dynamical critical phenomena and noise driven hydrodynamics [41, 42, 60]. An expansion in powers of $\lambda$ in combination with a momentum shell integration yields to leading order in $d-2$ the DRG equation $d g / d l=\beta(g)$, with beta-function $\beta(g)=(2-d) g+$ const. $g^{2}$ [61, 62]. Here $g=\lambda^{2} \Delta / \nu^{3}$ is the effective coupling strength and $l$ the logarithmic scale parameter. Below the lower critical dimension $d=2$ the DRG flow is towards a strong coupling fixed point with scaling exponents $\zeta=1 / 2$ and $z=3 / 2$ in $d=1$. Above $d=2$ a kinetic phase transition line delimits a strong coupling regime from a weak coupling regime. In the strong coupling regime the DRG flow is towards a still poorly understood strong coupling fixed point with unknown scaling exponents and scaling function. In the weak coupling regime the DRG flow is towards a weak coupling fixed point with scaling exponents $z=2$ and $\zeta=(2-d) / 2$. On the transition line $z=2$ and $\zeta=0$, see e.g. Ref. [33]. In Fig. 1 we have depicted the scaling properties in a plot of the coupling strength $g$ versus the dimension $d$.

We note two further properties of the KPZ equation which are also relevant in a scaling context. Firstly, subject to a Galilean transformation the equation is invariant provided we add a constant slope to the height field $h$ and adjust the drift $F$ accordingly, i.e.,

$$
\begin{aligned}
& \mathbf{r} \rightarrow \mathbf{r}-\lambda \mathbf{u}^{0} t \\
& h \rightarrow h+\mathbf{u}^{0} \cdot \mathbf{r}, \\
& F \rightarrow F+(\lambda / 2) \mathbf{u}^{0} \cdot \mathbf{u}^{0}
\end{aligned}
$$

note that the slope field $\mathbf{u}$ and diffusive field $w$ transform according to $\mathbf{u} \rightarrow \mathbf{u}+\mathbf{u}^{0}$ and $w \rightarrow w \exp \left[(\lambda / 2 \nu) \mathbf{u}^{0} \cdot \mathbf{r}\right]$, respectively. From a simple scaling argument and also following 
from the DRG analysis the Galilean invariance implies the scaling law

$$
\zeta+z=2
$$

relating the roughness and dynamic scaling exponents. The Galilean invariance is a fundamental dynamical symmetry specific to the KPZ equation, delimiting the KPZ universality class. Secondly, a fluctuation dissipation theorem is operational in 1D in the sense that the stationary Fokker-Planck equation associated with the KPZ equation admits the explicit solution [39, 63]

$$
P_{0}(h) \propto \exp \left[-\frac{\nu}{\Delta} \int d x(\nabla h)^{2}\right] .
$$

The Gaussian form of the distribution shows that the slope $u=\nabla h$ fluctuations are uncorrelated and that the height field $h=\int^{x} u d x^{\prime}$ performs a random walk in $x$. Note also that the distribution is independent of the non-linear growth parameter $\lambda$.

\section{Weak noise method}

Whereas the DRG approach, based on an asymptotic expansion about the critical dimension $d=2$, deals with the long time - large distance scaling properties of the KPZ equation, the asymptotic weak noise approach addresses the stochastic growth morphology or many body aspects. The weak noise or canonical phase space method focuses on the noise strength $\Delta$ as the relevant parameter in the problem. In the absence of noise for $\eta=0$ or $\Delta=0$ the morphology of the deterministic KPZ equation decays subject to a transient pattern formation; in 1D corresponding to cusps connected by parabolic segments [38]. In the presence of even weak noise the KPZ equation is eventually driven into a stationary stochastic state; the cross-over time diverging in the limit of vanishing noise. In this sense the noise strength $\Delta$ is a singular parameter and a weak noise approach asymptotic in $\Delta$.

The weak noise canonical phase space approach is implemented by applying an eikonal or WKB approximation to the Fokker-Planck equation associated with the Langevin equation. Viewing the Fokker-Planck equation as an imaginary time Schödinger equation the scheme is equivalent to the well-known WKB or semi-classical approximation in quantum mechanics, where the wave function $\Psi$ is related to the classical action $S$ by $\Psi \propto \exp [i S / \hbar], \hbar$ being the Planck constant. In quantum mechanics the quantum fluctuations characterized by orbitals 
are then in the correspondence limit $\hbar \rightarrow 0$ replaced by orbits as solutions to the classical equations of motion following from the action $S$.

In the weak noise approach the point of departure is a general Langevin equation of the form

$$
\begin{aligned}
& \frac{d x}{d t}=-F(x)+\eta(t), \\
& \langle\eta \eta\rangle(t)=\Delta \delta(t)
\end{aligned}
$$

for simplicity we consider a single random variable $x(t)$; for the more general case see e.g. Refs. [33, 64]. Here $F(x)$ is a general non-linear drift, and $\eta$ an additive white noise correlated with strength $\Delta$. In order to implement the weak noise approximation we consider the equivalent Fokker-Planck equation for the distribution $P(x, t)$ :

$$
\Delta \frac{\partial P}{\partial t}=\frac{1}{2} \Delta^{2} \frac{\partial^{2} P}{\partial x^{2}}+\Delta \frac{\partial}{\partial x}(F P) .
$$

Interpreting $\Delta \partial / \partial x$ as a momentum operator, $P$ as an effective wave function, and $\Delta$ as an effective Planck constant, Eq. (2.15) has the form of an imaginary time Schrödinger equation. Consequently, in the weak noise limit it is suggestive to introduce the WKB or eikonal approximation [65]

$$
P(x, T) \propto \exp \left[-\frac{S(x, T)}{\Delta}\right] .
$$

To leading order in $\Delta$ the action $S$ then obeys a principle of least action $\delta S=0$ as expressed by the Hamilton-Jacobi equation $\partial S / \partial t+H(x, p)=0$ with associated canonical momentum $p=\partial S / \partial x[66,67]$. The Hamiltonian (energy) takes the form

$$
H=\frac{1}{2} p^{2}-p F=\frac{1}{2} p(p-2 F)
$$

yielding the coupled Hamilton equations of motion

$$
\begin{aligned}
& \frac{d x}{d t}=-F+p, \\
& \frac{d p}{d t}=p \frac{d F}{d x} .
\end{aligned}
$$

Finally, the action associated with an orbit from $x_{i}$ to $x$ in the transition time $T$ is given by

$$
S(x, T)=\int_{x_{i}, 0}^{x, T} d t\left[p \frac{d x}{d t}-H\right],
$$


or inserting the equations of motion for $x$

$$
S(x, T)=\frac{1}{2} \int_{x_{i}, 0}^{x, T} d t p^{2} .
$$

The issue of solving the stochastic Langevin equation (2.13) or, equivalently, the deterministic Fokker-Planck equation (2.15) in the weak noise limit $\Delta \rightarrow 0$ is then replaced by, as first step, solving the coupled equations of motion (2.18,2.19) for an orbit from an initial configuration $x_{i}$ at time $t=0$ to a final configuration $x$ at time $t=T$. In the next step we evaluate the action $S$ associated with the orbit and infer from the WKB ansatz (2.16) the transition probability for the specific transition. We note that the noise in Eq. (2.13) has been replaced by the canonical momentum $p$ and that $p$ is a dependent variable which has to be chosen in accordance with the initial and final values of $x$ and the imposed transition time $T$.

In a phase space representation the zero-energy manifolds $p=0$ and $p=2 F$ intersecting at a hyperbolic saddle point play an important role in determining the long time stationary distribution $P_{0}(x)=\lim _{T \rightarrow \infty} P(x, T)$. Initially an orbit from $x_{i}$ to $x$ moves along the the transient zero-energy manifold $p=0$ towards the saddle point. This part of the orbit represents the transient motion. As time progresses the orbits bends away from the saddle point and is attracted to the stationary submanifold $p=2 F$. This part of the orbit corresponds to the cross-over to a stationary random motion. In the limit of a long transition time the orbit from $x_{i}$ to $x$ passes close to the saddle point, at which the large waiting time ensures the Markoff property. In Fig. 2 we have sketched the $\{x, p\}$ phase space showing the zero-energy submanifolds, the saddle point and an orbit from $x_{i}$ to $x$ in transition time $T$.

\section{Growth modes}

In the KPZ case the weak noise scheme is most easily implemented for the $\mathrm{CH}$ equation (2.6) driven by multiplicative noise. This requires an extension of the weak noise method discussed in Ref. [32, 33]. Introducing the wavenumber parameters

$$
\begin{aligned}
& k=\left(\lambda F / 2 \nu^{2}\right)^{1 / 2}, \\
& k_{0}=\lambda / 2 \nu
\end{aligned}
$$


setting inverse length scales, we find the weak noise Hamiltonian

$$
H=\int d^{d} x\left(p\left[\nu \nabla^{2}-\nu k^{2}\right] w+(1 / 2) k_{0}^{2}(w p)^{2}\right),
$$

and associated field equations

$$
\begin{aligned}
& \frac{\partial w}{\partial t}=\nu\left[\nabla^{2} w-k^{2} w\right]+k_{0}^{2} w^{2} p \\
& \frac{\partial p}{\partial t}=-\nu\left[\nabla^{2} p-k^{2} p\right]-k_{0}^{2} p^{2} w
\end{aligned}
$$

determining orbits in a $\{w, p\}$ phase space. Likewise, one infers the action

$$
S(w, T)=\frac{1}{2} k_{0}^{2} \int^{w, T} d^{d} x d t(w p)^{2},
$$

yielding the transition probability to leading asymptotic order in $\Delta$

$$
P(w, T) \propto \exp \left[-\frac{S(w, T)}{\Delta}\right] ;
$$

note that on the $p=0$ manifold Eq. (2.25) reduces to the deterministic $\mathrm{CH}$ equation for $\eta=0$.

The equations of motion $(2.25,2.26)$ serve two purposes. On the one hand, a solution or orbit in phase space from an initial configuration $w_{i}(\mathbf{r})$ at time $t=0$ to a final configuration $w(\mathbf{r})$ at time $t=T$ with $p$ as an adjusted noise field yields an action $S$ and thus a contribution to the transition probability $P(w, T)$. Secondly, the solution $w(\mathbf{r}, t)$ interpreted as a classical orbit also provides a growth morphology for the $\mathrm{CH}$ equation. The deterministic growth or evolution of the diffusive field $w$ then corresponds to a growth morphology for the KPZ equation by means of the inverse Cole-Hopf transformation

$$
h=\left(1 / k_{0}\right) \ln w .
$$

Likewise, the transition probability $P(h, T)$ is given by

$$
P(h, T)=\int \prod_{\mathbf{r}} d w \delta\left(h-\left(1 / k_{0}\right) \log w\right) P(w, T) .
$$

The growth morphology follows from the coupled nonlinear field equations (2.25) 2.26$)$. Owing to the negative diffusion coefficient the equations are numerically unstable, see Ref. [68], however, searching for localized instanton or soliton type solutions we note that on the $p=0$ and $p=\nu w$ submanifolds the static equations reduce to the static diffusion equation and 
the static nonlinear Schrödinger equation, well known in the context of dark solitons in Bose condensed atomic gasses [68]

$$
\begin{aligned}
& \nabla^{2} w=k^{2} w \\
& \nabla^{2} w=k^{2} w-k_{0}^{2} w^{3} .
\end{aligned}
$$

\section{E. Domain walls in 1D}

In 1D Eqs. (2.31,2.32) admit the static solutions $w_{ \pm} \propto \cosh ^{ \pm 1} k x$ for the diffusive field $w$. These modes correspond to cusps in the height field, $h_{ \pm}= \pm\left(1 / k_{0}\right) \ln (\cosh k x)$, and to static domain walls or solitons in the local slope field:

$$
u_{ \pm}(x)= \pm \frac{k}{k_{0}} \tanh k x
$$

The right hand domain wall, $u_{+}(x)=\left(k / k_{0}\right) \tanh k x$, is associated with the $p=0$ manifold and carries zero energy and zero action. This mode is the well-known viscosity-smoothed shock wave solution of the static noiseless Burgers equation $\nu \nabla^{2} u+\lambda u \nabla u=0$, as easily seen by inspection [44]. The left hand domain wall, $u_{-}(x)=-\left(k / k_{0}\right) \tanh k x$, lives on the $p=\nu w$ manifold and carries a finite action

$$
S=\frac{8 \nu^{2} k^{3}}{3 k_{0}^{2}} T
$$

the static domain walls are depicted in Fig. 3. By applying the Galilean transformation $(2.8,2.10)$ the static domain walls can be boosted to a finite propagation velocity and we obtain the moving domain walls or growth modes

$$
u_{ \pm}(x, t)= \pm \frac{k}{k_{0}} \tanh k\left(x-\lambda u^{0} t\right)-u_{0}
$$

The propagating domain walls form the basic building blocks in the construction of a growth morphology. Considering a dilute gas of non overlapping growth modes of different amplitudes or "charges" $k_{i}$, where a positive charge corresponds to a right hand domain wall and 
a negative charge to a left hand domain wall, we obtain the global solution [33]

$$
\begin{aligned}
& u(x, t)=\frac{1}{k_{0}} \sum_{i} k_{i} \tanh \left|k_{i}\right|\left(x-x_{i}(t)\right), \\
& h(x, t)=\frac{1}{k_{0}} \sum_{i} \frac{k_{i}}{\left|k_{i}\right|} \ln \left(\cosh \left|k_{i}\right|\left(x-x_{i}(t)\right)\right), \\
& x_{i}(t)=\int_{0}^{t} v_{i}\left(t^{\prime}\right) d t^{\prime}+x_{i}^{0}, \\
& v_{i}(t)=-\frac{\lambda}{k_{0}} \sum_{l \neq i} k_{l} \tanh \left|k_{i}\right|\left(x_{i}(t)-x_{l}(t)\right) ;
\end{aligned}
$$

note that the neutrality condition $\sum_{i} k_{i}=0$ ensures that the interface is flat at infinity. This condition, however, allows for an offset in $h$ corresponding to propagating facets.

The interpretation of the time dependent growth morphology is straightforward. For a dilute gas of growth modes the velocities adjust to constant values after a transient period and the growth modes move ballistically. Moreover, superimposed on the growth modes is a gas of diffusive modes following from a linear analysis of the equation of motion about the domain wall solutions, see Ref. [69]. In Fig. 4 we have depicted a three domain wall growth configuration composed of interconnected propagating domain walls, two right hand domain walls and one left hand domain wall. We also show the resulting morphology in the height field corresponding to moving steps or facets.

In order to make contact with the stochastic interpretation we prepare the interface in a specific initial state $h(x, 0)$ characterized by a gas of growth modes plus diffusive background. By also assigning an appropriate noise field $p(x, 0)$ corresponding to the nucleation of growth modes this configuration propagates ballistically forward in time to a specific finite configuration $h(x, T)$. Only the left hand domain walls corresponding to negative charges carry an action. For a dilute domain wall gas, ignoring the diffusive contribution, this action is additive, i.e.,

$$
S=\frac{8 \nu^{2} T}{3 k_{0}^{2}} \sum_{k_{i}<0}\left|k_{i}\right|^{3},
$$

yielding the transition probability

$$
P(h, T) \propto \exp \left[-\frac{S(h, T)}{\Delta}\right] .
$$

For illustration consider the two-domain wall configuration depicted in Fig. 5, This pair 
mode has the form

$$
u(x, t)=\frac{k}{k_{0}}\left[u_{+}\left(x-v t-x_{1}\right)+u_{-}\left(x-v t-x_{2}\right)\right]
$$

and moves according to the domain wall matching condition following from the Galilean symmetry with the velocity $v=-\lambda k / k_{0}$. Since the pair mode in the slope $u$ corresponds to a moving step in $h$ the propagation across the system either subject to periodic or bouncing boundary condition corresponds to adding a layer to the interface; the mode thus corresponds to a specific growth situation. The mode moves ballistically with an action given by Eq. (2.34) carried by the left hand domain wall; note that the right hand domain wall partner

carries zero action. In time $T$ the mode moves the distance $L=v T$ and we obtain the transition probability

$$
P(L, T) \propto \exp \left[-\frac{4 \nu}{3 \lambda^{2} \Delta} \frac{L^{3}}{T^{2}}\right] .
$$

We conclude that the step in $h$ performs a random walk with mean square displacement

$$
<L^{2}>\propto\left(\lambda^{2} \Delta / \nu\right)^{2 / 3} T^{2 / z}
$$

characterized by the dynamical exponent $z=3 / 2$. This result is in accordance with established scaling results for the KPZ equation in 1D, see e.g. Ref. [39]. The facet in the height field corresponding to the pair growth mode thus performs superdiffusion [70].

\section{THE MINIMUM ACTION METHOD}

In this section we discuss the basis for the minimum action method characterized by the Freidlin-Wentzel action and the connection to equivalent formulations in non equilibrium physics.

\section{A. Freidlin Wentzel scheme}

The point of departure for the Freidlin-Wentzel (FW) scheme is a generic Langevin equation for a set of stochastic variables, $\left\{x_{n}\right\}$, driven by additive white Gaussian noise

$$
\frac{d x_{n}}{d t}=-F_{n}\left(\left\{x_{m}\right\}\right)+\eta_{n}(t)
$$


where the noise is distributed according to

$$
\left.P\left(\left\{\eta_{n}\right)\right\}, T\right) \propto \exp \left[-\frac{1}{2 \Delta} \int_{0}^{T} d t \sum_{n} \eta_{n}(t)^{2}\right] .
$$

A heuristic derivation of the Freidlin-Wentzel action, the basis for the minimum action method, follows in the weak noise limit by simply replacing the noise $\eta_{n}$ in Eq. (3.2) by $d x_{n} / d t+F_{n}$ yielding

$$
P\left(\left\{x_{n}\right\}, T\right) \propto \exp \left[-\frac{1}{2 \Delta} \int_{0}^{T} d t \sum_{n}\left(\frac{d x_{n}}{d t}+F_{n}\right)^{2}\right] .
$$

Expressing $P\left(\left\{x_{n}\right\}, T\right)$ in the WKB form

$$
P\left(\left\{x_{n}\right\}, T\right) \propto \exp \left[-\frac{S_{\mathrm{FW}}}{\Delta}\right]
$$

we readily identify the Freidlin-Wentzel action

$$
S_{\mathrm{FW}}=\frac{1}{2} \int_{0}^{T} d t \sum_{n}\left[\frac{d x_{n}}{d t}+F_{n}\right]^{2}
$$

for rigorous details see Refs. [10, 28].

The minimum action method then corresponds to minimizing the action $S_{\mathrm{FW}}$ subject to

an initial condition $\left\{x_{n}(0)\right\}$, a final condition $\left\{x_{n}(T)\right\}$, and a given transition time $T$. The method thus identifies the minimum action path in the "action landscape". The method works both for gradient systems where $F_{n}$ can be derived from a free energy,

$$
F_{n}=\nabla_{n} \Phi
$$

including e.g. the GL case and non-gradient systems like the KPZ equation.

\section{B. Martin Siggia Rose scheme}

The Martin-Siggia-Rose (MSR) scheme [16, 17, 18, 19, 20, 21] also takes as its starting point the Langevin equation (3.1). For simplicity we consider, however, only a single stochastic variable $x(t)$. For the transition probability $P(x, T)$ we have by definition

$$
P(x, T)=\langle\delta(x-x(T))\rangle_{\eta}
$$

where we average over the noise $\eta$ driving the Langevin equation. Incorporating the Langevin equation determining the evolution of $x(t)$ as a delta function constraint, averaging over the 
noise $\eta$ according to Eq. (3.2), noting that the change of variable from $d x / d t$ to $x$ yields the Jacobian $J=\exp \left[(1 / 2) \int d t d F / d x\right]$, and finally setting $p \rightarrow p / \Delta$ we obtain the functional phase space integral [71]

$$
P(x, T) \propto \int \prod_{t} d x d p \delta(x-x(T)) \exp \left[-i \frac{S_{\mathrm{MSR}}}{\Delta}\right],
$$

where the MSR action is given by

$$
S_{\mathrm{MSR}}=\int d t\left[p \frac{d x}{d t}-H\right]
$$

with MSR Hamiltonian

$$
H_{\mathrm{MSR}}=-\frac{i}{2} p^{2}-p F+\frac{i \Delta}{2} \frac{d F}{d x}
$$

Since $p$ appears quadratically it can be eliminated by a Gaussian integration and we arrive at the configuration space path integral

$$
P(x, T) \propto \int \prod_{t} d x \delta(x-x(T)) \exp \left[-\frac{S}{\Delta}\right],
$$

with action

$$
S=\frac{1}{2} \int d t\left[\left(\frac{d x}{d t}+F\right)^{2}-\Delta \frac{d F}{d x}\right] .
$$

We note that this form holds for arbitrary noise strength. The path integral is a formal solution of the Fokker-Planck equation. In the aymptotic weak noise limit $\Delta \rightarrow 0$ only the saddle point in the path integral contributes. Ignoring the Jacobian contribution $\Delta d F / d x$ we recover the FW result in Eq. (3.3) in the case of one variable.

\section{Quantum analogue and phase space method}

Contact with the Fokker-Planck equation (2.15) is easily achieved by noting that Eq. (3.8) has the form of a Feynmann path integral with Planck constant $\Delta$ [71, 72, 73]. Introducing the momentum operator $\hat{p}=-i \Delta d / d x$ the quantum Hamiltonian operator takes the form

$$
\hat{H}=\frac{i}{2} \Delta^{2} \frac{d^{2}}{d x^{2}}+\left(i \Delta \frac{d F}{d x}\right)_{\text {order }}+\frac{i \Delta}{2} \frac{d F}{d x},
$$


where the ordering in the term $(i \Delta d F / d x)_{\text {order }}$ remains to be fixed. Choosing the symmetrical Weyl ordering $(d F / d x)_{\text {order }}=(1 / 2)(F d / d x+d F / d x)$ the Schrödinger equation associated with $\hat{H}$,

$$
i \Delta \frac{\partial P}{\partial t}=\hat{H} P
$$

then reduces to the Fokker-Planck equation (2.15). Finally, formally rotating $p, p \rightarrow i p$, we obtain a real path integral representation for $P$,

$$
P(x, T) \propto \int \prod_{t} d x d p \delta(x-x(T)) \exp \left[-\frac{S}{\Delta}\right],
$$

with action

$$
S=\int d t\left[p \frac{d x}{d t}-H\right]
$$

and Hamiltonian

$$
H=\frac{1}{2} p^{2}-p F+\frac{\Delta}{2} \frac{d F}{d x} .
$$

In the weak noise limit $\Delta \rightarrow 0$ the Jacobian contribution in Eq. (3.17) can be ignored and only the saddle point in Eq. (3.15) contributes, corresponding to a principle of least action $\delta S=0$. In this way we recover the results in Section II C. We note that the canonical phase space method is completely equivalent to the Freidlin-Wentzel scheme for the extremal orbits. In fact inserting Eq. (3.1) for one variable, $d x / d t=-F+p$ in Eq. (2.21) we obtain $S=(1 / 2) \int d t p^{2}$ which is the Freidlin-Wentzel action. The advantage of the phase space method is the introduction of the canonically conjugate momentum $p$, representing the noise, as an additional variable. This allows for a phase space representation of the numerical results obtained by a numerical optimization of the Freidlin-Wentzel action. In Fig. 6] we have in a $x t$ plot depicted the paths in configuration space from an initial configuration $x_{i}$ at time $t=0$ to a final configuration $x$ at time $t=T$. We have also shown the extremal path which dominates the path integral in the limit $\Delta \rightarrow 0$.

\section{MINIMUM ACTION METHOD FOR THE KPZ EQUATION}

In this section we apply the minimum action method to the KPZ equation and set up the numerical scheme. For the KPZ equation the FW action has the form

$$
S=\frac{1}{2} \int \boldsymbol{d} \mathbf{r} d t\left(\frac{\partial h}{\partial t}-\nu \nabla^{2} h-\frac{\lambda}{2}(\nabla h)^{2}+F\right)^{2} .
$$


In order to find the optimal switching path from an initial configuration $h_{\text {init }}(\mathbf{r})$ at time $t=0$ to a final configuration $h_{\text {fin }}(\mathbf{r})$ at time $T$ we minimize the action (4.1) subject to the constraints:

$$
h(\mathbf{r}, 0)=h_{\text {init }}(\mathbf{r}), \quad h(\mathbf{r}, T)=h_{\text {fin }}(\mathbf{r}) .
$$

We first discretize the action functional using finite differences, then minimize the discretized action functional using the limited memory Broyden-Fletcher-Goldfarb-Shanno (BFGS) method. The BFGS is an efficient quasi-Newton method for large scale optiminization problems [74]. It is an iterative method; at each iteration, it only requires the input of the action $S$ and the associated gradient $\delta S / \delta h(\mathbf{r}, t)$. The minimization is constrained by appropriate Dirichlet boundary conditions in space, $h(\mathbf{r}, t)=h_{\mathcal{B}}(\mathbf{r})$ for $\mathbf{r}$ on boundary $\mathcal{B}$, and initial and final boundary conditions in time, $h(\mathbf{r}, 0)=h_{\text {init }}(\mathbf{r})$ and $h(\mathbf{r}, T)=h_{\text {fin }}(\mathbf{r})$

In the following we consider the $1 \mathrm{D}$ case. We confine the system to a $1 \mathrm{D}$ interval of size $L$ and the switching path to the time interval $T$. In the space-time domain $[0, L] \times[0, T]$ we introduce a mesh with sizes $\Delta x=L / I$ and $\Delta t=T / J$ and define the grid points $\left(x_{i}, t_{j}\right)$

$$
\begin{array}{ll}
x_{i}=i \Delta x & i=0, \cdots I, \\
t_{j}=j \Delta t & j=0, \cdots J .
\end{array}
$$

The numerical approximation to $h\left(x_{i}, t_{j}\right)$ is denoted by $H_{i j}$. In order to simplify the expression we introduce the momentum or the noise field

$$
p(x, t)=\frac{\partial h}{\partial t}-\nu \frac{\partial^{2} h}{\partial x^{2}}-\frac{\lambda}{2}\left(\frac{\partial h}{\partial x}\right)^{2}+F,
$$

and express the action in the form

$$
S(h)=\frac{1}{2} \int_{0}^{T} d t \int_{0}^{L} d x p^{2}(x, t) .
$$

Using the trapezoidal rule to discretize the integral in space and the midpoint rule to compute the temporal integral we obtain

$$
S(H)=\frac{1}{2} \Delta x \Delta t \sum_{i=1}^{I-1} \sum_{j=1}^{J} P_{i j}^{2}
$$


where the discretized version of the noise field is

$$
\begin{aligned}
& P_{i j}=\frac{H_{i j}-H_{i, j-1}}{\Delta t}+F \\
& -\nu \frac{H_{i+1, j}+H_{i-1, j}-2 H_{i j}}{2(\Delta x)^{2}} \\
& -\nu \frac{H_{i+1, j-1}+H_{i-1, j-1}-2 H_{i, j-1}}{2(\Delta x)^{2}} \\
& -\frac{\lambda}{2} \frac{\left(H_{i+1, j}-H_{i-1, j}+H_{i+1, j-1}-H_{i-1, j-1}\right)^{2}}{16(\Delta x)^{2}} .
\end{aligned}
$$

For the discretized boundary condition we have

$$
\begin{aligned}
& H_{0 j}=H_{1}, \quad H_{I j}=H_{2} \text { for } j=0, \cdots J \\
& H_{i 0}=h_{\text {init }}\left(x_{i}\right), \quad H_{i J}=h_{\text {fin }}\left(x_{i}\right) \text { for } i=0, \cdots I,
\end{aligned}
$$

where $H_{1}$ and $H_{2}$ denotes the boundary values. For an offset in the height profile we have $H_{1} \neq H_{2}$. The BFGS method also requires the gradient of the action, whose discrete version is given by

$$
\begin{aligned}
& \frac{\partial S}{\partial H_{i j}}=\Delta x \Delta t\left(\frac{P_{i j}-P_{i, j+1}}{\Delta t}\right. \\
& -\nu \frac{P_{i+1, j}+P_{i-1, j}-2 P_{i j}}{2(\Delta x)^{2}} \\
& -\nu \frac{P_{i+1, j+1}+P_{i-1, j+1}-2 P_{i, j+1}}{2(\Delta x)^{2}} \\
& -\frac{\lambda}{2} \frac{\left(H_{i j}-H_{i-2, j}+H_{i, j+1}-H_{i-2, j+1}\right) P_{i-1, j+1}}{8(\Delta x)^{2}} \\
& +\frac{\lambda}{2} \frac{\left(H_{i+2, j}-H_{i j}+H_{i+2, j+1}-H_{i, j+1}\right) P_{i+1, j+1}}{8(\Delta x)^{2}} \\
& -\frac{\lambda}{2} \frac{\left(H_{i, j-1}-H_{i-2, j-1}+H_{i j}-H_{i-2, j}\right) P_{i-1, j}}{8(\Delta x)^{2}} \\
& \left.+\frac{\lambda}{2} \frac{\left(H_{i+2, j-1}-H_{i, j-1}+H_{i+2, j}-H_{i j}\right) P_{i+1, j}}{8(\Delta x)^{2}}\right) .
\end{aligned}
$$

The numerical optimization is set up by choosing an initial pathway interpolating between the initial and final configurations $h_{\text {init }}$ and $h_{\text {fin }}$ subject to the chosen boundary conditions. Provided that the initial pathway lies in the domain of the appropriate minimum of $S$ the BFGS method then through successive steps finds the minimum action and outputs the weak noise pathway from $h_{\text {init }}(x)$ to $h_{\text {fin }}(x)$ in a given transition time $T$. 


\section{NUMERICAL RESULTS IN 1D}

In this section we discuss various switching scenarios for the KPZ equation in $1 \mathrm{D}$. As parameter values we choose for the viscosity $\nu=1$ and for the non-linear growth parameter $\lambda=2$. These values yield the inverse length scale $k_{0}=1$. The parameter $k$ is then given by $k=\sqrt{F}$ where $F$ is the imposed drift. We, moreover, consider a system of size $L=1$.

We consider the switching scenario in $1 \mathrm{D}$ from an initial state $h(x, 0)=-h_{0}$ to a final state $h(x, T)=h_{0}$. This transition corresponds to adding a layer of thickness $2 h_{0}$ to the interface. At the boundaries $x=0$ and $x=L$ we set $h=0$, i.e., $H_{1}=H_{2}=0$. In order to match the initial profile $h_{\text {init }}(x)=-h_{0}$ to the boundary condition we use the cusp solutions in Eq. (2.37), $h_{ \pm}(x)= \pm\left(1 / k_{0}\right) \ln |\cosh k x|$ and set $h(x, 0)=h_{L}(x)+h_{R}(x)$, where

$$
\begin{aligned}
& h_{L}(x)=-\frac{1}{k_{0}} \ln \left|\frac{\cosh k\left(x-x_{1}\right)}{\cosh k\left(x-x_{1}-\delta\right)}\right|, \\
& h_{R}(x)=\frac{1}{k_{0}} \ln \left|\frac{\cosh k\left(x-x_{2}\right)}{\cosh k\left(x-x_{2}-\delta\right)}\right| .
\end{aligned}
$$

Setting $x_{1} \sim 0$ and $x_{2} \sim L-\delta$ and choosing $\delta=h_{0} k_{0} / 2 k$ the initial profile satisfies the boundary conditions and approach the interface value $-h_{0}$ in the bulk; note that the slope of the steps is given by $1 / k$. In our simulation we choose $x_{1}=0.1, x_{2}=0.8, \delta=0.1$. Likewise, the final configuration at time $t=T$ is given by $h(x, T)=-h_{L}(x)-h_{R}(x)$. To ensure a steep step corresponding to a short healing length we choose the drift $F=625$ corresponding to $k=25$. With this choice $h_{0}=2 k \delta / k_{0}=5$. For the initial path, we use the linear interpolation between $h(x, 0)$ and $h(x, T): h(x, t)=(1-t / T) h(x, 0)+(t / T) h(x, T)$. Finally, we have chosen a $200 \times 200$ set of $x t$ grid points.

In Figs. 7 - 10 we show switching scenarios for the transition times $T=0.1, T=0.03$, $T=0.01$, and $T=0.001$. We depict both the height profiles $h(x, t)$, the slope profiles $u(x, t)$, and the associated noise profiles $p(x, t)$. In Figs. 11, 14 we depict the associated squared noise field or action density in a space-time plot.

The height profiles presented for the initial and final configurations $h_{\text {init }}$ and $h_{\text {fin }}$ and for some characteristic intermediate times show that the transition or switch in time $T$ is effectuated by the ballistic propagation of steps or facets across the system. The corresponding slope profiles demonstrate that the steps can be interpreted in terms of a gas of domain walls with opposite parity, i.e., right hand and left hand domain walls. The motion of a single step in $h$ is thus associated with a pair of co-moving domain walls in $u$ moving across the 


\begin{tabular}{|c|c|}
\hline Transition time $T$ & Switching action $S$ \\
\hline 0.100 & $2.57 \times 10^{3}$ \\
0.030 & $2.56 \times 10^{3}$ \\
0.010 & $3.12 \times 10^{3}$ \\
0.001 & $1.95 \times 10^{4}$ \\
\hline
\end{tabular}

TABLE I: The switching actions $S(T)$ associated with the transition times $T=$ 0.100, 0.030, 0.010, 0.001.

system. The dependent noise field $p$ is associated with the nucleation of domain walls. Since the right-hand domain wall is a solution of the deterministic Burgers equation it carries no dynamical attributes and the associated noise field vanishes, unlike the "noise-induced" left-hand domain wall which is associated with a noise field and carries an action. In Table I we show the actions associated with the transitions and in Figs. 15 the action as a function of the transition time for the various scenarios.

\section{DISCUSSION AND INTERPRETATION}

In this section we interpret the numerical results in 1D using the weak noise canonical phase method. As discussed in Sec. III the phase space method is completely equivalent to the minimum action method.

\section{A. Waiting time transition for $T=0.1$}

In terms of the switching dynamics $T=0.1$ corresponds to a long-time transition. In Fig. 7 we show snapshots of $h, u$, and $p$ at times $t=0.0,0.05,0.0875,0.0925,0.1$; in Fig. 11 we depict the squared noise field or space-time action density. In the initial stage of the transition, from $t=0$ to about $t=0.075$, the constant height field makes a transition to a trough (convex cusp) compatible with the boundary conditions $h=0$. This configuration corresponds to a static right hand domain wall in the slope $u$. After a long waiting time in this configuration (until about $t=0.075$ ) domain walls in $u$ nucleate at the boundaries and a pair of domain walls then move across the system from left to right. In the height field this mode corresponds to the motion of a facet or step. The trough in $h$ is filled in and 
eventually at time $T$ the final configuration $h_{\text {fin }}$ is reached. The noise field associated with the waiting time configuration vanishes since it corresponds to a right hand domain wall. However, in the final stage of the transition the noise field develops corresponding to the nucleation of the left hand domain wall.

This switching scenario is in accordance with the phase space interpretation generically represented in Fig. 2. For a long time transition the orbit comes close to the saddle point corresponding to $p=0$. In the slope field this implies a configuration given by the right hand domain wall $u=\left(k / k_{0}\right) \tanh k x$ yielding the cusp in Fig. 7a. After a long waiting time in the vicinity of the saddle point the orbit eventually wanders off along the stationary manifold towards the final configuration. This part of the orbit in phase phase associated with a finite noise field corresponds to the propagation of the step in $h$, associated with the domain wall pair in $u$.

The action can also be estimated qualitatively. For a single left-hand domain wall the action is given by Eq. (2.34), $S_{\mathrm{dw}}=(8 / 3) \nu^{2}\left(k^{3} / k_{0}^{2}\right) T$. Inserting $\nu=1, k_{0}=1$, and $k=25$ we obtain $S_{\mathrm{dw}}=41667 \times T$. However, owing to the waiting time only the last $p \neq 0$ part of the orbit contributes to $S_{\mathrm{dw}}$. Estimating the effective transition time to be $T \sim 0.05$ we obtain an action of order $S_{\mathrm{dw}} \sim 2000$ which should be compared with the numerical value from Table I, $S_{\text {num }}=2567$. The discrepancy can be accounted for by the finite nucleation action at the boundaries and also the finite system size effect.

\section{B. Intermediate time transitions, $T=0.03$ and $T=0.01$}

In Figs 8 and 9 we have depicted switching scenarios at transition times $T=0.03$ and $T=$ 0.01 for the height, slope and noise. In Fig. 8 we show snapshots along the pathway at times $t=0.0,0.015,0.0188,0.0225,0.03$ and in Fig. 9 at times $t=0.0,0.0025,0.005,0.0075,0.01$. In Figs. 12 and 13 we depict the squared noise field or space-time action density. Since the imposed transition time is shorter compared to the previous case the waiting time is shortened. The transition again is driven by the nucleation and subsequent propagation of domain walls. In the case $T=0.03$ domain walls in $u$ are nucleated at the edges and the pair propagates across the system with a positive velocity similar to the waiting time case. In the case $T=0.01$ the shorter transition time favors the nucleation of a domain wall in $u$ at the center. This domain wall subsequently breaks up into two pairs of domain wall 
moving toward the edges. In the height profile this scenario corresponds to the nucleation of a tip which subsequently broadens to a plateau effectuating the transition.

This switching scenario is again heuristically in agreement with the phase space interpretation in Fig. 2, For an intermediate time transition the orbit in phase space bends off towards the stationary finite $p$ manifold at an earlier stage in order to effectuate the transition in the shorter time interval available.

The action based on Eq. (2.34) is again of the same order of magnitude as the numerical results listed in Table I. We note that the shorter transition time requires a larger domain wall velocity $v \propto k_{i}$, where $k_{i}$ is the charge of the particular domain wall. Since the action scales with $k_{i}^{3}$ this effect compensates in the action for the smaller $T$. For an infinite system the imposed drift $F \propto k^{2}$ in the KPZ equation is related to the domain wall charges $k_{i}$ by the

relationship $k=\sum_{i} k_{i}$. Due to finite size effects this relation cannot be used directly in the present context. However, we still conclude that the imposed $k$ does not fix the individual charges. The domain wall amplitudes and thus velocities are determined by the transition scenario.

\section{Short time transition, $T=0.001$}

In Fig. 10 we show the switching scenario for the transition times $T=0.001$ for the height, slope and noise. In Fig. 10 we show the snapshots along the pathway at times $t=0.0,0.00025,0.00075,0.001$. In Fig. 14 we depict the squared noise field or space-time action density. In the short time regime it is more advantageous to nucleate multiple domain wall pairs in the slope field, corresponding to multiple steps of facets in the height field.

\section{Switching action}

In Fig. 15 we depict the action $S(T)$ as a function of the transition time $T$ for five transition scenarios. The circles correspond to the transition pathways we discussed earlier and shown in Fig. 7[10 for $T=0.01,0.03,0.01,0.001$; the remaining pathways (not shown) involve one nucleation at the center and one nucleation from the boundary. The plot clearly indicates that more domain wall pairs, yielding a lower action, are nucleated at shorter transition times. 
This relationship can be accounted for by the following considerations. For a single domain wall pair propagating across the system the associated action is given by $S_{1}=$ $S_{\text {nucl }}+A(L / T)^{3} T$. Here $S_{\text {nucl }}$ is the nucleation action associated with the left handed domain wall. The second term follows from Eq. (2.34), where we note that the velocity $v=L / T$ scales with the amplitude $k ; A$ is a constant which we do not have to specify further. In the case of a transition effectuated by the nucleation and transition of two domain wall pairs we have correspondingly for the action, $S_{2}=2 S_{\text {nucl }}+2 A(L / 2 T)^{3} T$, where we note that the domain wall pair only propagate half the distance. In the general case of $n$ domain wall pairs we obtain the expression

$$
S_{n}=n S_{\text {nucl }}+A \frac{L^{3}}{n^{2} T^{2}} .
$$

In Fig. [16 we have depicted $S(T)$ versus $T$ for different values of $n$, which shows that the multi domain wall transitions have lower action at shorter time. This result follows from the competition between the nucleation action and the action associated with the propagation and is in qualitative agreement with the numerical results shown in Fig. 15.

\section{TRANSITION PATHWAYS IN 2D}

In 2D the weak noise approach yields elementary spherically symmetric growth modes. In terms of the diffusive field $w$ the diffusion equation (2.31), $\nabla^{2} w=k^{2} w$, has the asymptotic growing solution $w_{+} \propto \exp (k r)$ for $r \gg 1 / k$ giving rise to the height field $h_{+}=\left(k / k_{0}\right) r$ and the slope field $\mathbf{u}_{+}=\left(k / k_{0}\right) \mathbf{r} / r$. Likewise, the non-linear Schrödinger equation (2.32), $\nabla^{2} w=k^{2} w-k_{0}^{2} w^{3}$, yields the decaying solution $w_{-} \propto \exp (-k r)$ and, correspondingly, $h_{-}=-\left(k / k_{0}\right) r$ and $\mathbf{u}_{-}=-\left(k / k_{0}\right) \mathbf{r} / r$. The height modes correspond to a tip (upward cone) and a dip (downward cone) in the interface profile, whereas the slope modes are outward and inward pointing vector fields of constant magnitude $k / k_{0}$, i.e., monopole fields. Like in the $1 \mathrm{D}$ case the static growth modes can be boosted to a finite propagation velocity and one can construct a dynamic growth morphology in terms of a dilute gas of monopoles in the slope field with superimposed diffusive modes. In a charge language the positive monopoles are solutions of the noiseless Burgers equation and carry no action, whereas the negative monopoles carry an action $S \propto\left(\nu^{2} T / k_{0}^{2}\right) k^{2}$. In order to model a pathway from $h_{\text {init }}$ at $t=0$ to $h_{\text {fin }}$ at $t=T$ one assigns a gas of monopoles representing $h_{\text {init }}$. 
With the appropriate assignment of the noise field corresponding to nucleation events this configuration will evolve in time to $h_{\mathrm{fin}}$. The total action associated with negative growth modes, using the WKB ansatz $P \propto \exp (-S / \Delta)$, then yields the transition probability for the kinetic pathway. Details of this procedure has been discussed at length in Ref. [33] and will not be reproduced here.

The minimum action method is easily extended to higher dimension generalizing the procedure in Sec. IV, Choosing the parameters $\nu=1, \lambda=2$, and a $100 \times 100 \times 100$ set of $x y t$ grid points and matching the height profile to the boundary values $h(\mathbf{r})=0$ by a 2D generalization of Eqs. (5.15.2), we have in Figs 17 and 18 depicted the 2D switching scenarios for the height field at transition times $T=0.02$ and $T=0.002$ from an initial plateau at $h=-5$ to a final plateau at $h=5$. In the case $T=0.02$ a single peak in $h$ is nucleated at the center of the plateau $h=-5$. The peak amplitude evolves in time and eventually flattens to the plateau at $h=5$. In the case $T=0.002$ the transition takes place subject to the nucleation of a regular pattern of growing cones in $h$ which eventually broadens and merge together. Like in the 1D case we note again that more peaks are nucleated at shorter transition times.

\section{SUMMARY AND CONCLUSION}

In the present paper we have applied the minimum action method based on the FreidlinWentzel scheme for rare events driven by weak noise to the KPZ equation for a growing interface. The KPZ equation is a non-gradient system and a characterization of kinetic pathways in a free energy landscape is not available, on the other hand, the pathways can be characterized as taking place in an action landscape. Correspondingly, the transition probabilities are characterized by the associated action of a specific pathway, unlike the free energy case for gradient systems where free energy considerations apply in the evaluation of the Arrhenius factor for the transition.

The minimum action method basically identifies the kinetic pathway in the action landscape by seeking a minimum of the action using an optimization technique. Once the minimum has been reached the method provides the kinetic pathway subject to given initial and final configurations combined with appropriate boundary conditions. We have conducted a detailed analysis of the 1D case and find that the pathways can be characterized by the 
nucleation and subsequent ballistic propagation of growth modes. These growth modes correspond to moving facets or steps in the KPZ height field and to moving domain walls in the slope field. We also find that the numerical results are in good qualitative agreement with the canonical phase analysis previously developed for the KPZ equation. We have, moreover, applied the minimum action method to the $2 \mathrm{D}$ case.

In conclusion, we believe that the minimum action method provides a new tool in analyzing the kinetics of spatially extended or field theoretical non-gradient systems like the KPZ equation studied here. The method supplements previous scaling analysis of the KPZ equation in focussing on the pattern formation or many body aspects of kinetic transitions in the weak noise limit.

\section{Acknowledgments}

The work of H. Fogedby has been supported by the Danish Natural Science Research Council under grant no. 95093801. The work of W. Ren is supported by NSF grant DMS0806401 and the Sloan fellowship. Discussions with A. Svane are gratefully acknowledged.

[1] P. Nelson, Biological Physics (Freeman, New York, 2003).

[2] P. M. Chaikin and T. C. Lubensky, Principles of Condensed Matter Physics (Cambridge University Press, Cambridge, 1995).

[3] M. C. Cross and P. C. Hohenberg, Rev. Mod. Phys 65, 851 (1994).

[4] A. L. Barabasi and H. E. Stanley, Fractal Concepts in Surface Growth (Cambridge University Press, Cambridge, 1995).

[5] P. L. Geissler, C. Dellago, D. Chandler, J. Hutter, and M. Parrinello, Science 291, 2121 (2001).

[6] R. Olender and R. Elber, J. Chem. Phys. 105, 9299 (1996).

[7] D. V. Berkov, J. Magn. Mag. Mat. 186, 199 (1998).

[8] R. H. Koch, G. Grinstein, G. A. Keefe, Y. Lu, P. L. Trouilloud, W. J. Gallagher, and S. S. P. Parkin, Phys. Rev. Lett. 84, 5419 (2000).

[9] C. Jaffe, S. D. Ross, M. W. Lo, J. Marsden, D. Farrelly, and T. Uzer, Phys. Rev. Lett. 89, 011101 (2002). 
[10] M. I. Freidlin and A. D. Wentzel, Random Perturbations of Dynamical Systems (2nd ed. Springer, New York, 1998).

[11] S. Machlup and L. Onsager, Phys. Rev. 91, 1512 (1953).

[12] L. Onsager and S. Machlup, Phys. Rev. 91, 1505 (1953).

[13] R. Graham and T. Tél, J. Stat. Phys. 35, 729 (1984).

[14] R. Graham and T. Tél, Phys. Rev. A 42, 4661 (1990).

[15] M. I. Dykman, Phys. Rev. A 42, 2020 (1990).

[16] P. C. Martin, E. D. Siggia, and H. A. Rose, Phys. Rev. A 8, 423 (1973).

[17] C. de Dominicis, Nuovo Cimento Lett. 12, 567 (1975).

[18] C. de Dominicis, J. Phys. (Paris), Colloq. 37, 247 (1976).

[19] R. Baussch, H. K. Janssen, and H. Wagner, Z. Phys. B 24, 113 (1976).

[20] H. K. Janssen, Z. Phys. B 23, 377 (1976).

[21] C. DeDominicis and L. Peliti, Phys. Rev. B 18, 353 (1978).

[22] P. G. Bolhuis, D. Chandler, C. Dellago, and P. Geissler, Ann. Rev. of Phys. Chem. 59, 291 (2002).

[23] W. Ren (2002), phD. Thesis (unpublished).

[24] W. E, W. Ren, and E. Vanden-Eijnden, Phys. Rev. B 66, 052301 (2002).

[25] W. E, W. Ren, and E. Vanden-Eijnden, J. Phys. Chem. B 109, 6688 (2005).

[26] W. Ren, E. Vanden-Eijnden, P. Maragakis, and W. E, J. Phys. Chem. 123, 134109 (2005).

[27] J. Garcia-Ojalvo and J. M. Sancho, Noise in Spatially Extended Systems (Springer, New York, 1999).

[28] W. E, W. Ren, and E. Vanden-Eijnden, Comm. Pure App. Math 57, 637 (2004).

[29] W. E, W. Ren, and E. Vanden-Eijnden, J. Appl. Phys. 93, 2275 (2003).

[30] H. C. Fogedby, J. Hertz, and A. Svane, Europhys. Lett 62, 795 (2003).

[31] H. C. Fogedby, J. Hertz, and A. Svane, Phys. Rev. E 70, 031105 (2004).

[32] H. C. Fogedby, Phys. Rev. Lett. 94, 195702 (2005).

[33] H. C. Fogedby, Phys. Rev. E 73, 031104 (2006).

[34] X. Zhou, W. Ren, and W. E, J. Phys. Chem. 128, 104111 (2008).

[35] M. Heymann and E. Vanden-Eijnden, Comm. Pure App. Math. 61, 1052 (2008).

[36] J. J. Binney, N. J. Dowrick, A. J. Fisher, and M. E. J. Newman, The Theory of Critical Phenomena (Clarendon Press, Oxford, 1992). 
[37] M. Kardar, G. Parisi, and Y. C. Zhang, Phys. Rev. Lett. 56, 889 (1986).

[38] E. Medina, T. Hwa, M. Kardar, and Y. C. Zhang, Phys. Rev. A 39, 3053 (1989).

[39] T. Halpin-Healy and Y. C. Zhang, Phys. Rep. 254, 215 (1995).

[40] J. Krug, Adv. Phys. 46, 139 (1997).

[41] D. Forster, D. R. Nelson, and M. J. Stephen, Phys. Rev. Lett. 36, 867 (1976).

[42] D. Forster, D. R. Nelson, and M. J. Stephen, Phys. Rev. A 16, 732 (1977).

[43] W. E and E. Vanden-Eijnden, Comm. Pure App. Math 53, 0825 (2000).

[44] W. A. Woyczynski, Burgers-KPZ Turbulence (Springer-Verlag, New York, 1998).

[45] J. M. Burgers, Proc. Roy. Neth. Acad. Soc. 32, 414, 643, 818 (1929).

[46] J. Burgers, The Nonlinear Diffusion Equation (Riedel, Boston, 1974).

[47] P. Saffman, Topics in Nonlinear Physics, edited by N.J. Zabusky (Springer, New York, 1968).

[48] E. Jackson, Perspectives of nonlinear dynamics (Cambridge University Press, Cambridge, 1990).

[49] G. B. Whitham, Nonlinear Waves (Wiley, New York, 1974).

[50] Y. Zeldovitch, Astron. Astrophys 8, 84 (1972).

[51] Shandarin and Y. B. Zeldovich, Rev. Mod. Phys 61, 185 (1989).

[52] J. D. Cole, Quart. Appl. Math. 9, 22 (1951).

[53] E. Hopf, Comm. Pure Appl. Math. 3, 201 (1950).

[54] M. Kardar, Nucl. Phys. B 290, 582 (1987).

[55] P. LeDoussal and K. J. Wiese, Phys. Rev. E 72, 035101(R) (2005).

[56] T. Halpin-Healy, Phys. Rev. E 58, R4096 (1998).

[57] F. Family, J. Phys. A 19, L441 (1986).

[58] F. Family, Physica A 168, 561 (1990).

[59] F. Family and T. Vicsek, J. Phys. A 18, L75 (1985).

[60] P. C. Hohenberg and B. I. Halperin, Rev. Mod. Phys 49, 435 (1977).

[61] K. J. Wiese, Phys. Rev. E 56, 5013 (1997).

[62] K. J. Wiese, J. Stat. Phys. 93, 143 (1998).

[63] D. A. Huse, C. L. Henley, and D. S. Fisher, Phys. Rev. Lett. 55, 2924 (1985).

[64] R. L. Stratonovich, Topics in the Theory of Random Noise (Gordon and Breach, New York, 1963).

[65] L. Landau and E. Lifshitz, Quantum Mechanics (Pergamon Press, Oxford, 1959). 
[66] H. Goldstein, Classical Mechanics (Addison-Wesley Publishing Company, Inc., Reading, Massachusetts, 1980).

[67] L. Landau and E. Lifshitz, Mechanics (Pergamon Press, Oxford, 1959).

[68] H. C. Fogedby and A. Brandenburg, Phys. Rev. E 66, 016604 (2002).

[69] H. C. Fogedby, Phys. Rev. E 68, 026132 (2003).

[70] J. Feder, Fractals (Plenum Press, New York, 1988).

[71] J. Zinn-Justin, Quantum Field Theory and Critical Phenomena (Oxford University Press, Oxford, 1989).

[72] R. Feynman and A. Hibbs, Quantum Mechanics and Path Integrals (McGraw-Hill Book Co., New York, 1965).

[73] A. Das, Field Theory - A Path Integral Approach (World Scientific, Singapore, 1993).

[74] J. Nocedal and S. J. Wright, Numerical Optimization (Springer-Verlag, Berlin, 1999). 


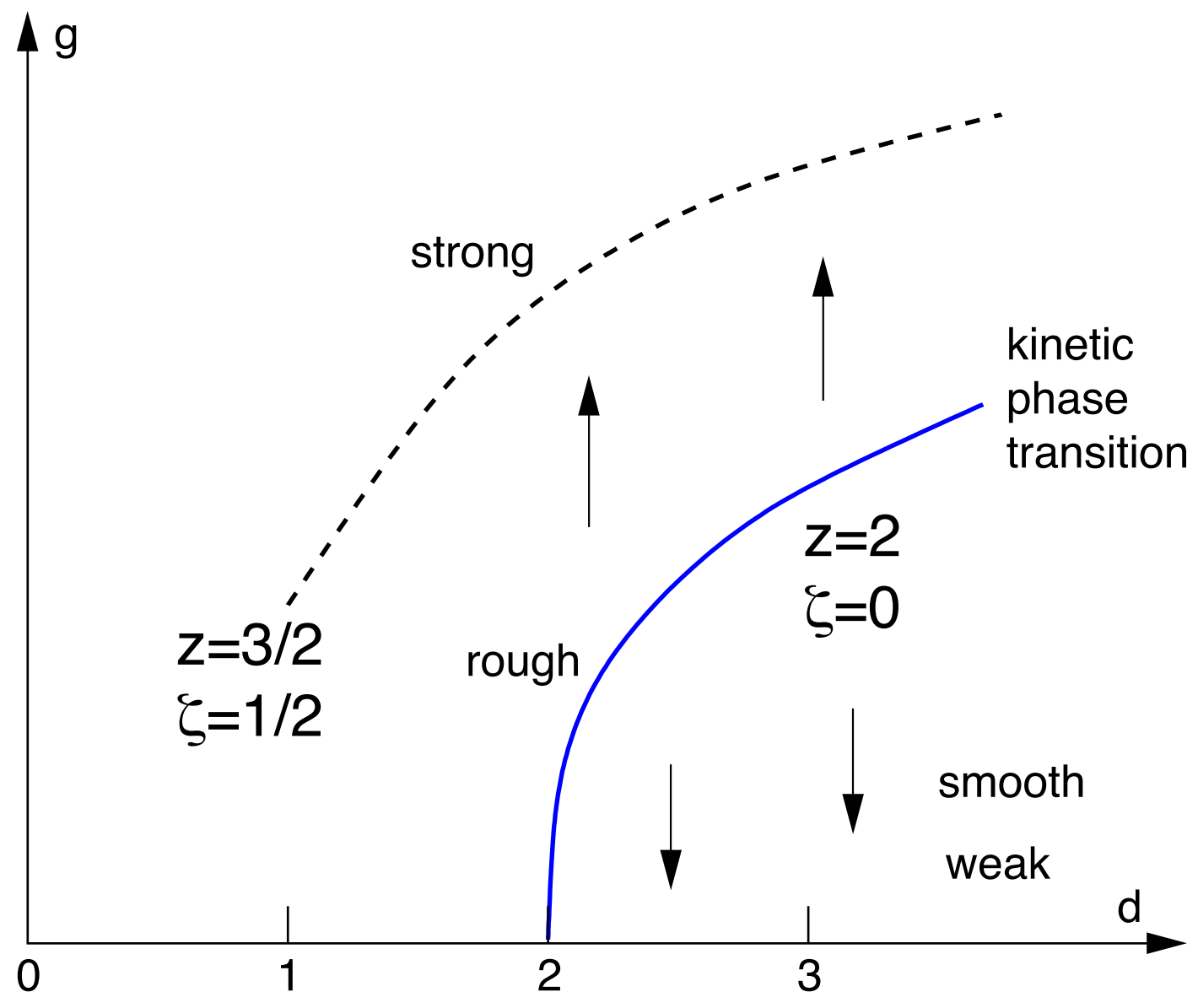

FIG. 1: DRG phase diagram for the KPZ equation to leading loop order in $d-2$. We plot the effective coupling strength $g=\lambda^{2} \Delta / \nu^{3}$ as a function of the dimension $d$. In $d=1$ the DRG flow is towards a strong coupling fixed point with scaling exponents $\zeta=1 / 2, z=3 / 2$. Above the lower critical dimension $d=2$ there is a kinetic transition line, delimiting a rough phase from a smooth phase. On the phase line $z=2$ and $\zeta=0$. The weak coupling smooth phase is characterized by $z=2$ and $\zeta=(2-d) / 2$. Above $d=1$ the scaling exponents in the strong coupling rough phase are unknown 


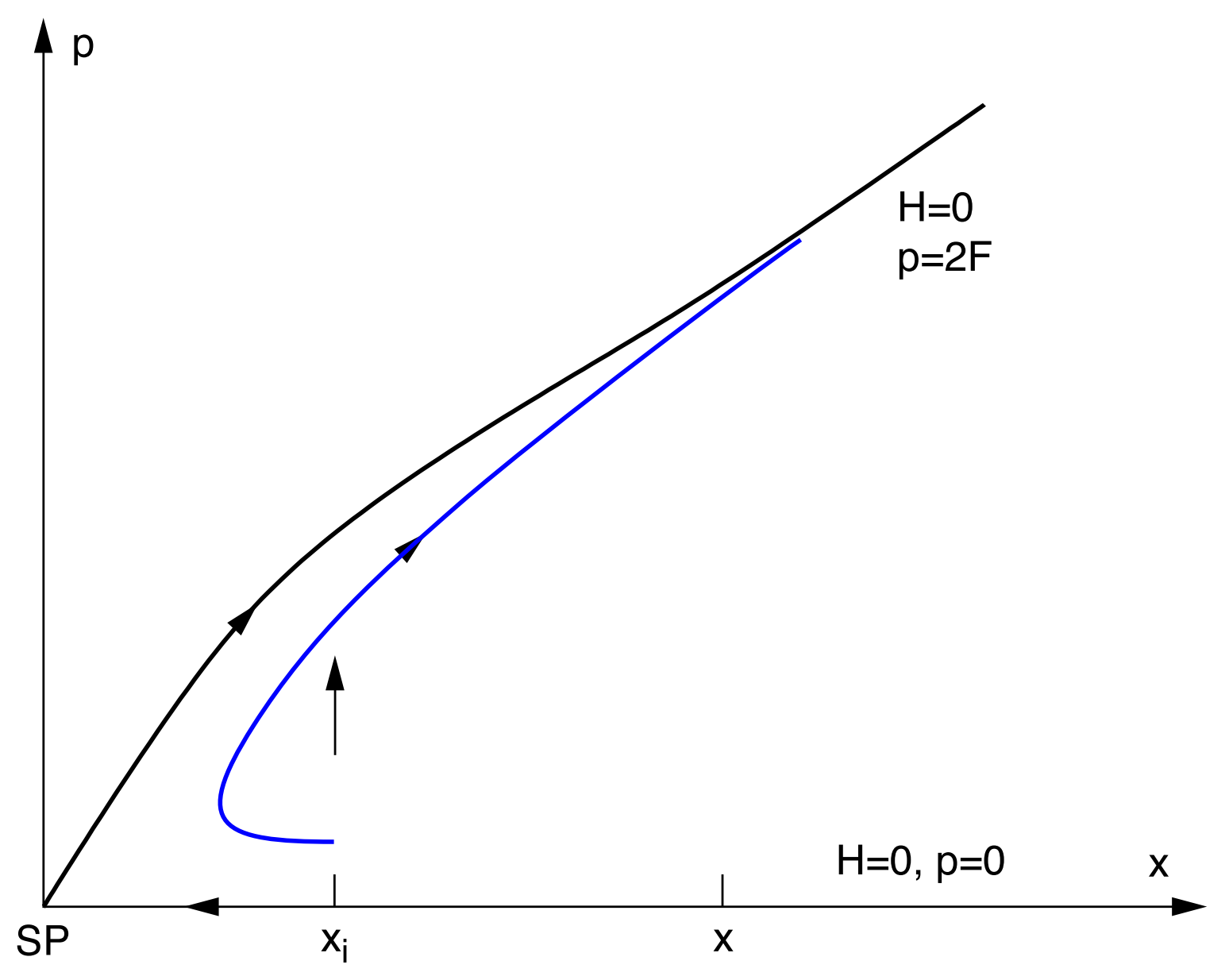

FIG. 2: Phase space representation of the weak noise method. We show the zero energy submanifold $p=0$ corresponding to the transient behavior and the submanifold $p=2 F$ determining the stationary distribution. The manifolds intersect in a hyperbolic saddle point (SP). The infinite waiting time at SP corresponds to the long time Markoff behavior. We depict a finite time orbit from $x_{i}$ to $x$ in transition time $T$ and an infinite time orbit passing through the saddle point. 

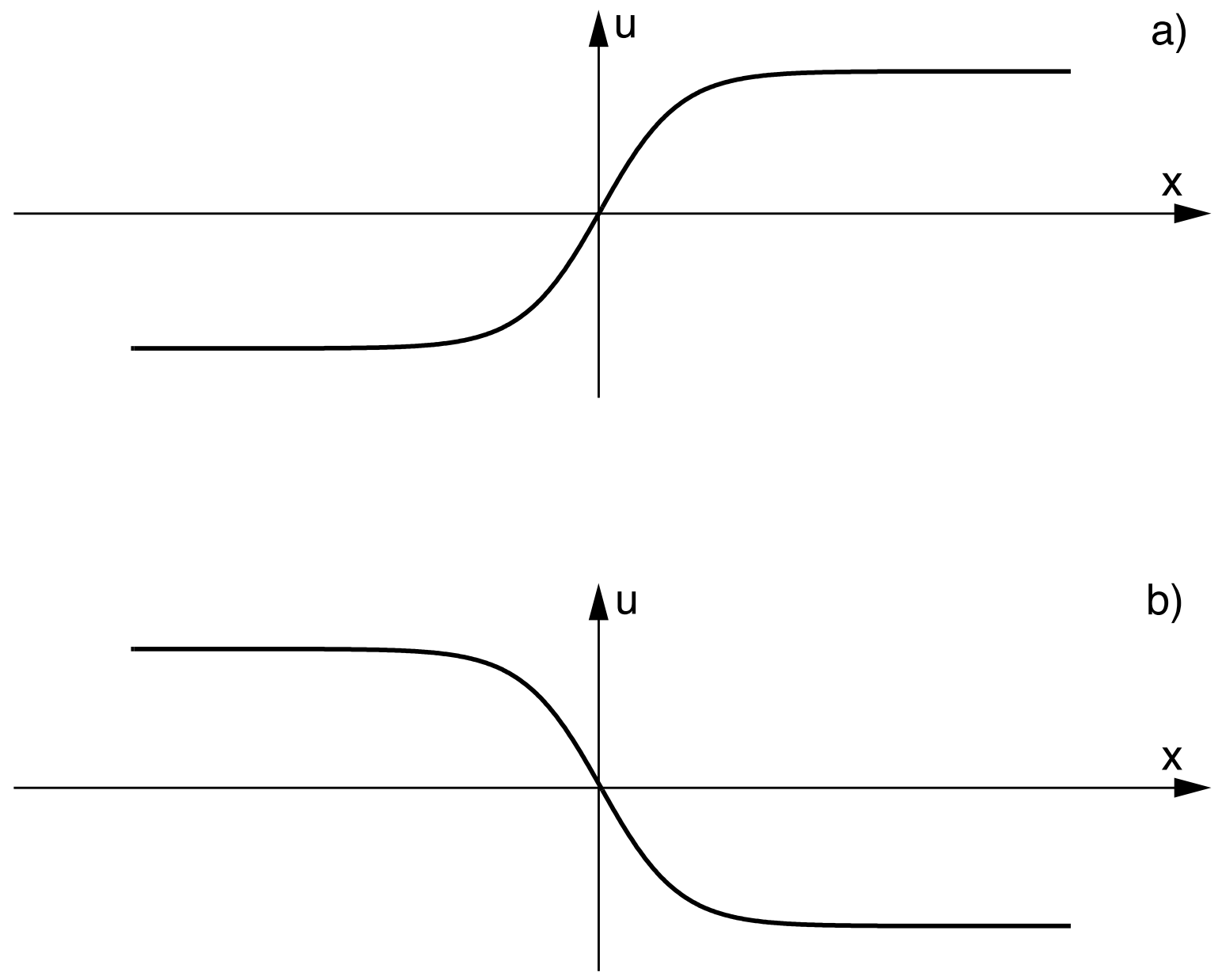

FIG. 3: We depict the static domain walls in the slope field corresponding to the solutions of the diffusion and non linear Schrödinger equations for the diffusive field . In a) we show the right hand domain wall. This domain wall carries vanishing action and is identical to the viscosity smoothed shock waves of the noiseless Burgers equation. In b) we show the noise induced left hand domain wall carrying a finite action. 

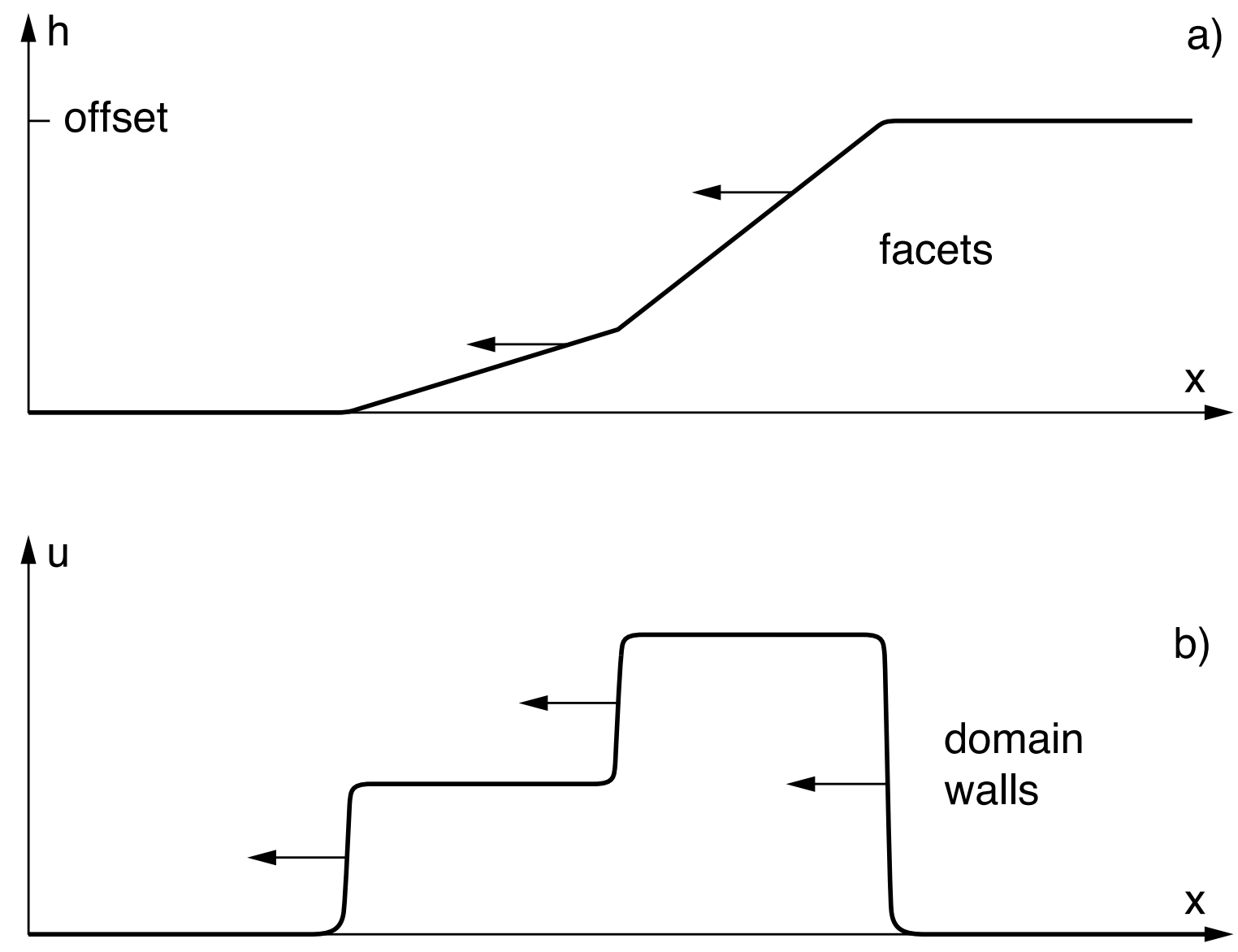

FIG. 4: We depict a three domain wall growth morphology. In b) we show the slope field composed of two right hand propagating domain walls and a single propagating left hand domain wall. In a) we show the corresponding moving facets in the height profile. Note that the charges of the domain walls add up to zero implying a flat interface at the edges, however, allowing for an offset 

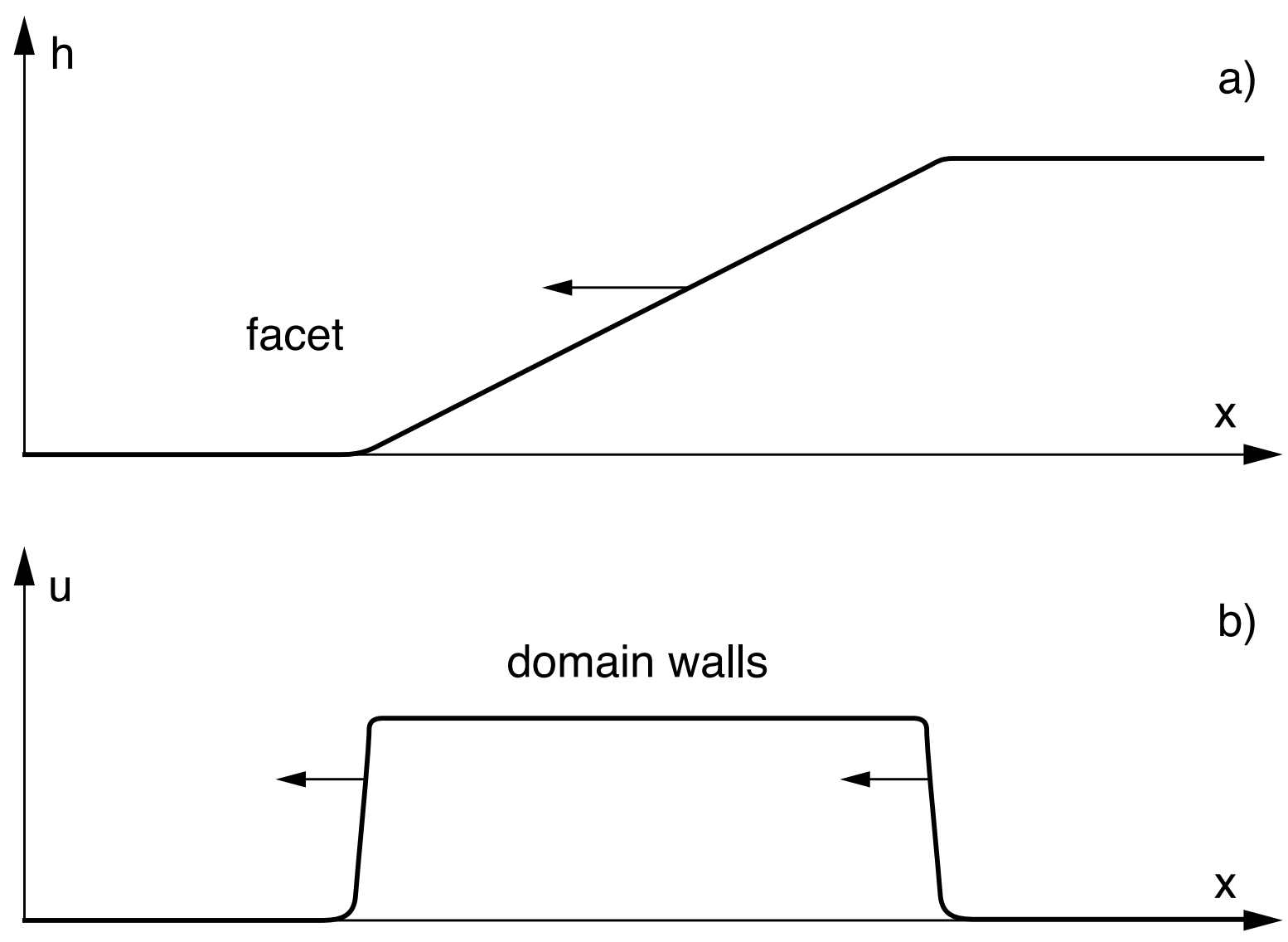

FIG. 5: In b) we show a co-moving two-domain wall configuration in the slope $u$. This pair mode corresponds to a moving step or facet in the height field $h$ depicted in a). The mode carries a finite action associated with the left hand domain wall. 


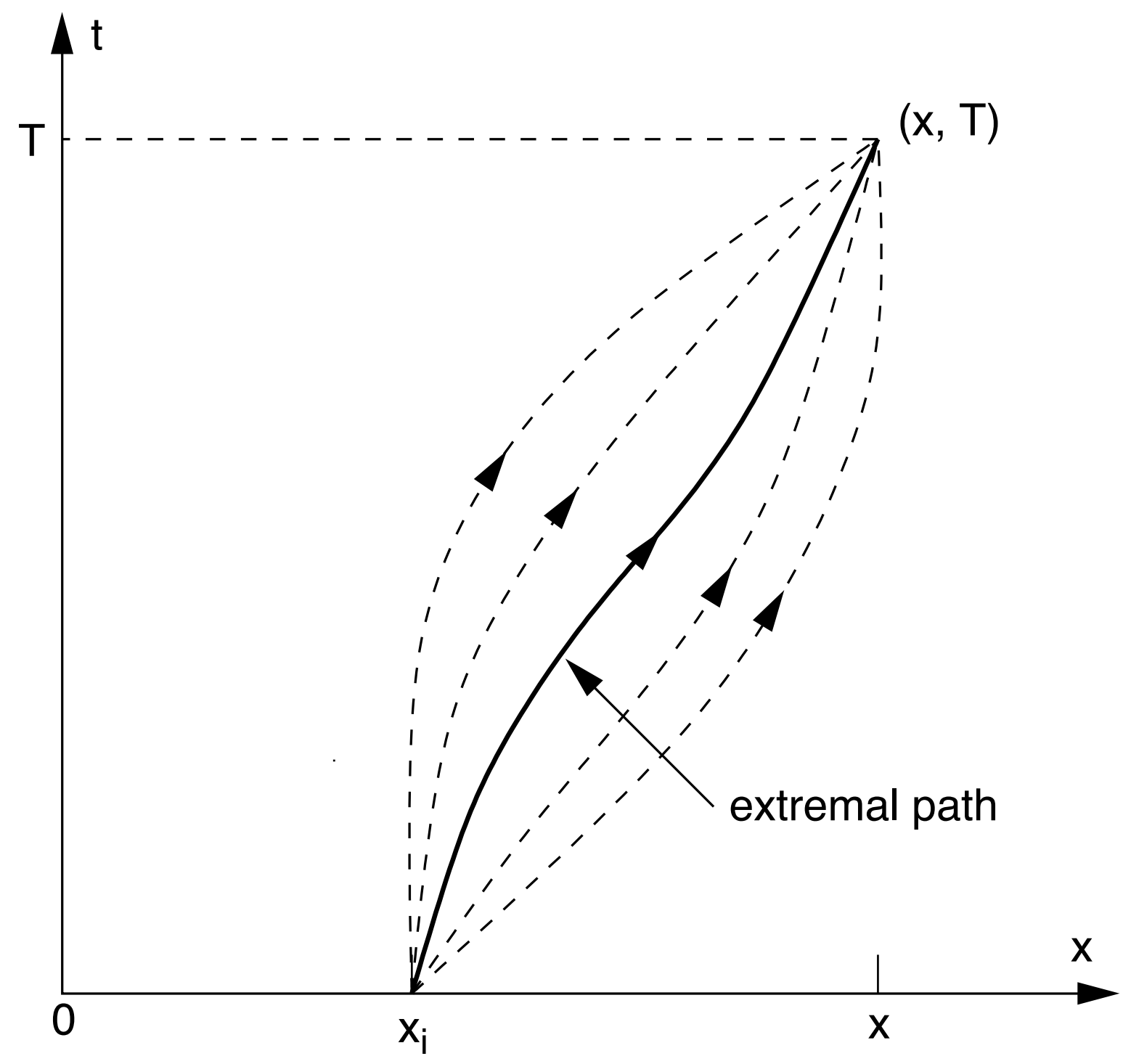

FIG. 6: We show paths (dashed) from an initial configuration $x_{i}$ at time $t=0$ to a final configuration $x$ at time $t=T$ contributing to the path integral. We also depict the extremal path (solid) dominating the path integral in the limit of weak noise. 

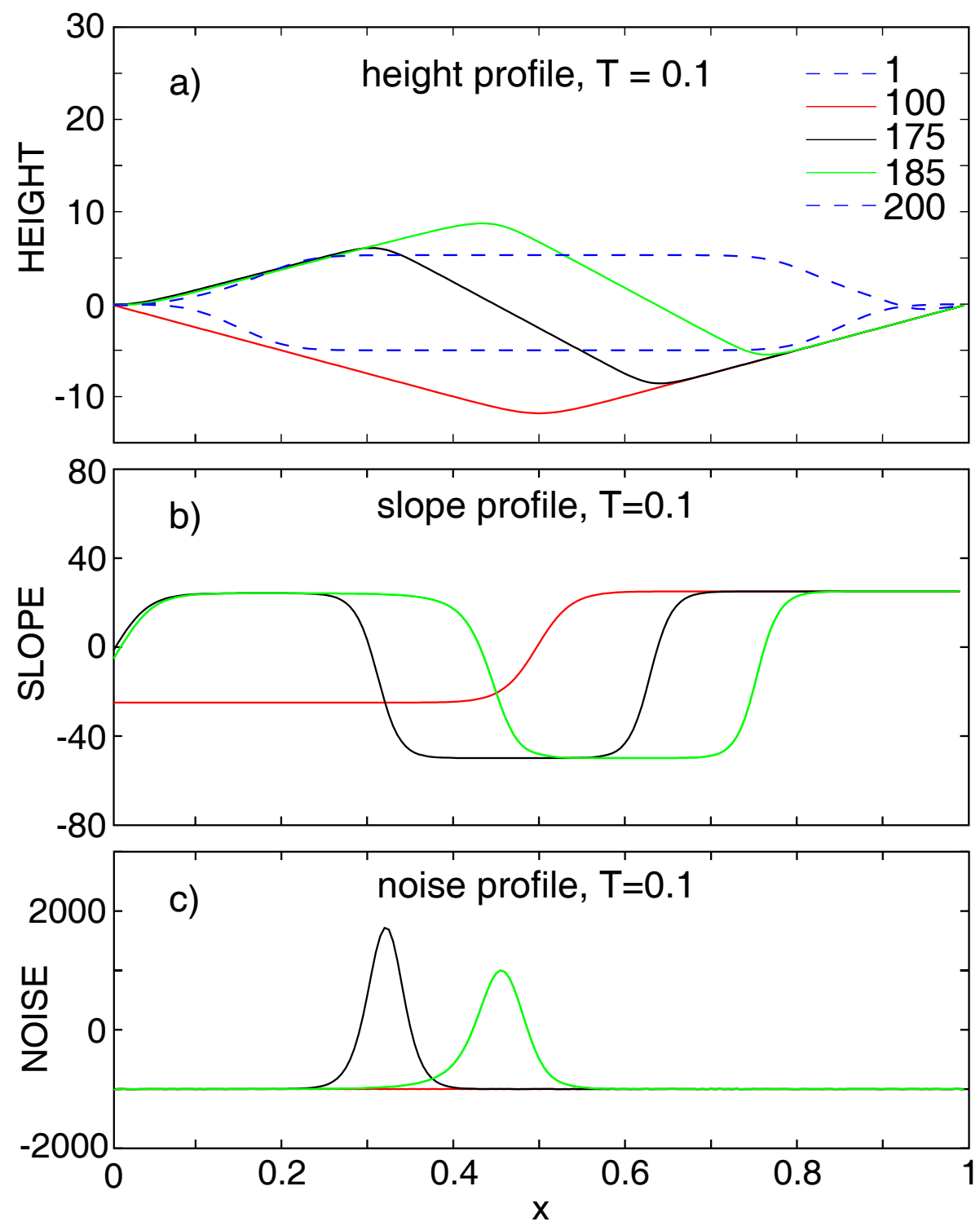

FIG. 7: We depict the transition scenario for transition time $T=0.1$. In a) we show the waiting time configuration and the propagating step in $h$, in b) the quasi static right hand domain wall and the propagating domain wall pair in $u$, and in c) the corresponding noise field associated with the propagating left hand domain wall (arbitrary units). 

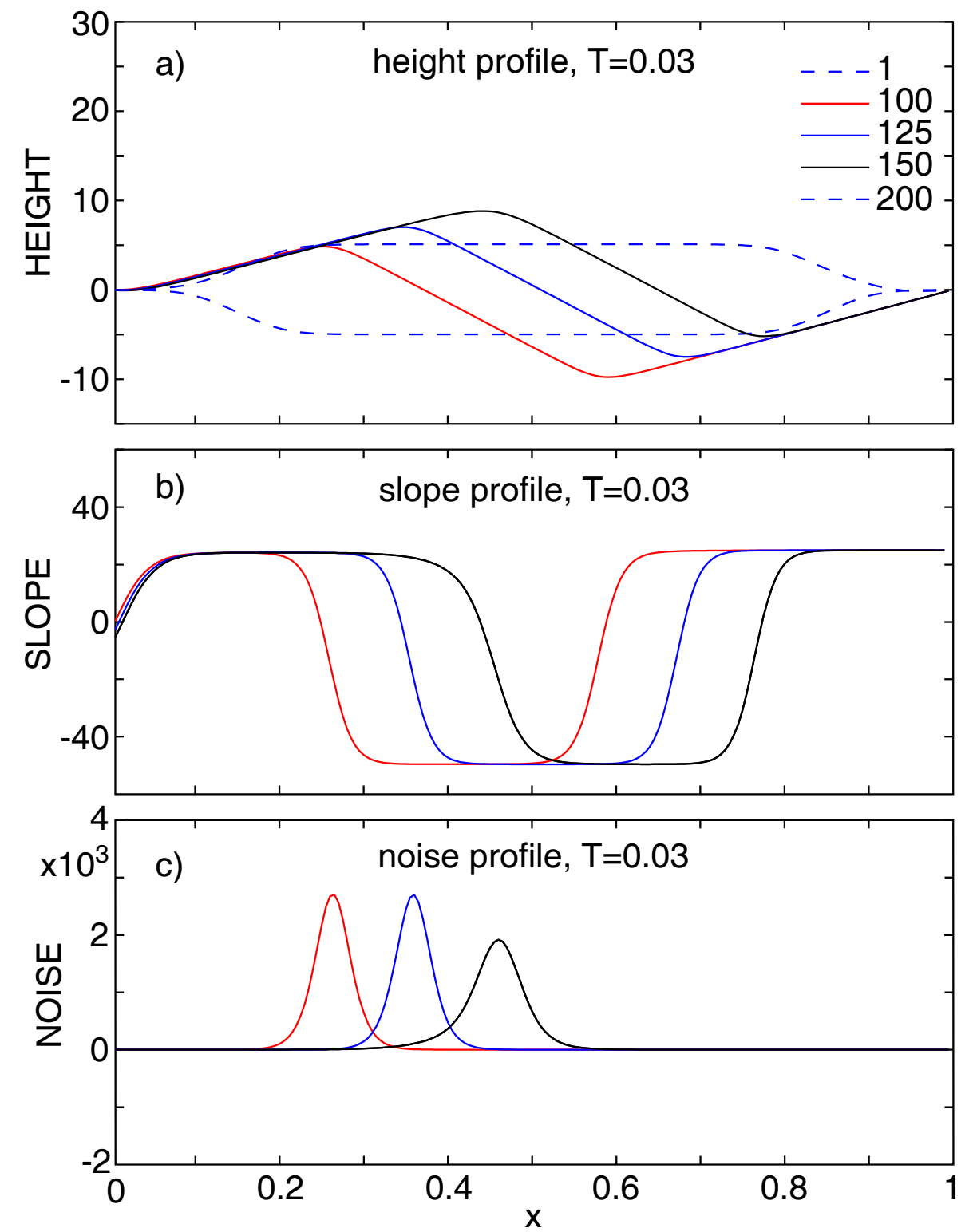

FIG. 8: We depict the transition scenario for transition time $T=0.03$. In a) we show the propagating step in $h$, in b) the propagating domain wall pair in $u$, and in c) the corresponding noise field associated with the propagating left hand domain wall (arbitrary units). 

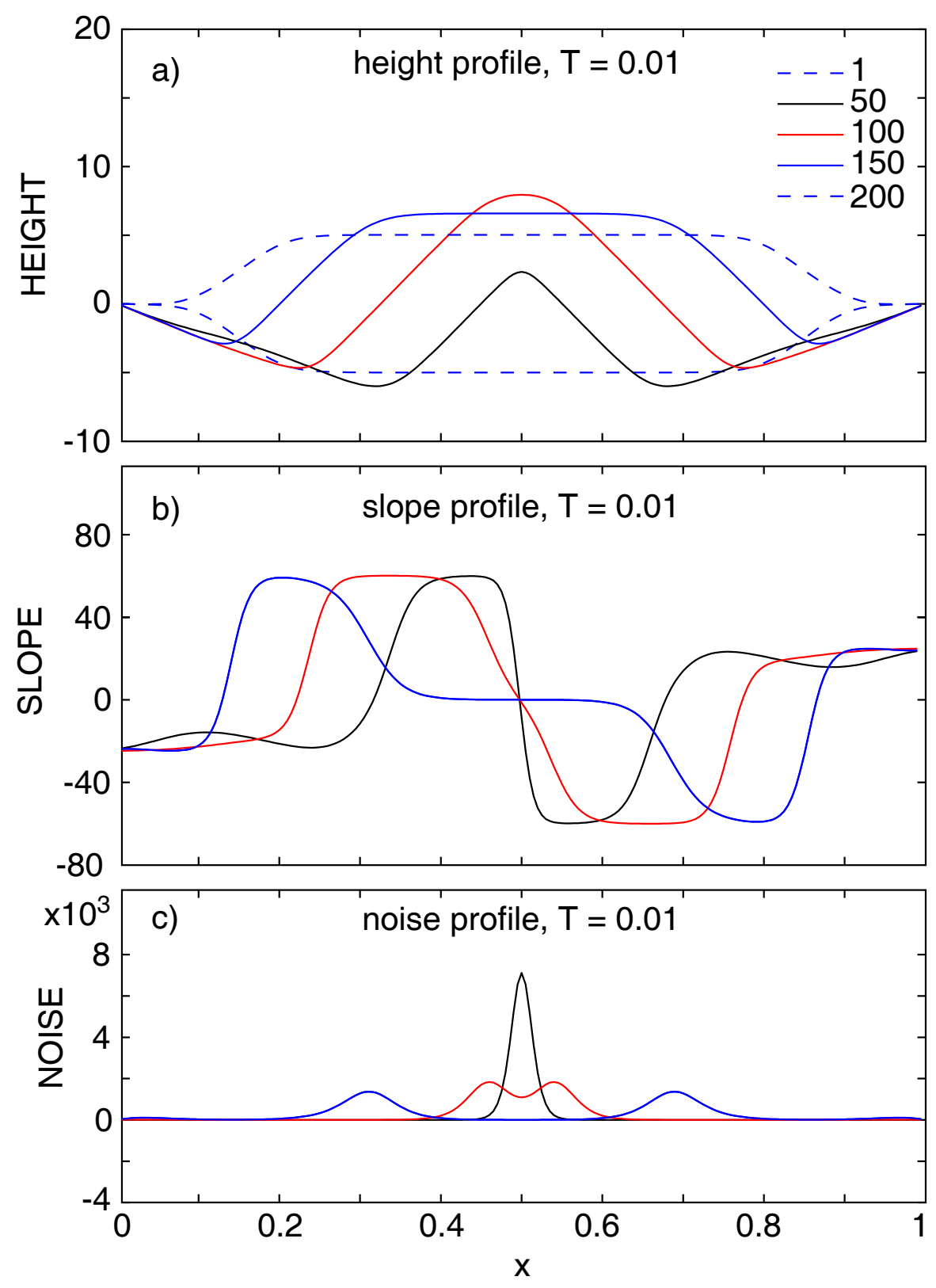

FIG. 9: We depict the transition scenario for transition time $T=0.01$. In a) we show the emerging plateau in $h$, in b) the left hand domain wall associated with the appearance of the peak in $h$ and the propagating domain wall pairs emerging from the center in $u$, and in c) the corresponding noise field associated with the nucleation and subsequent propagation from the center (arbitrary units). 

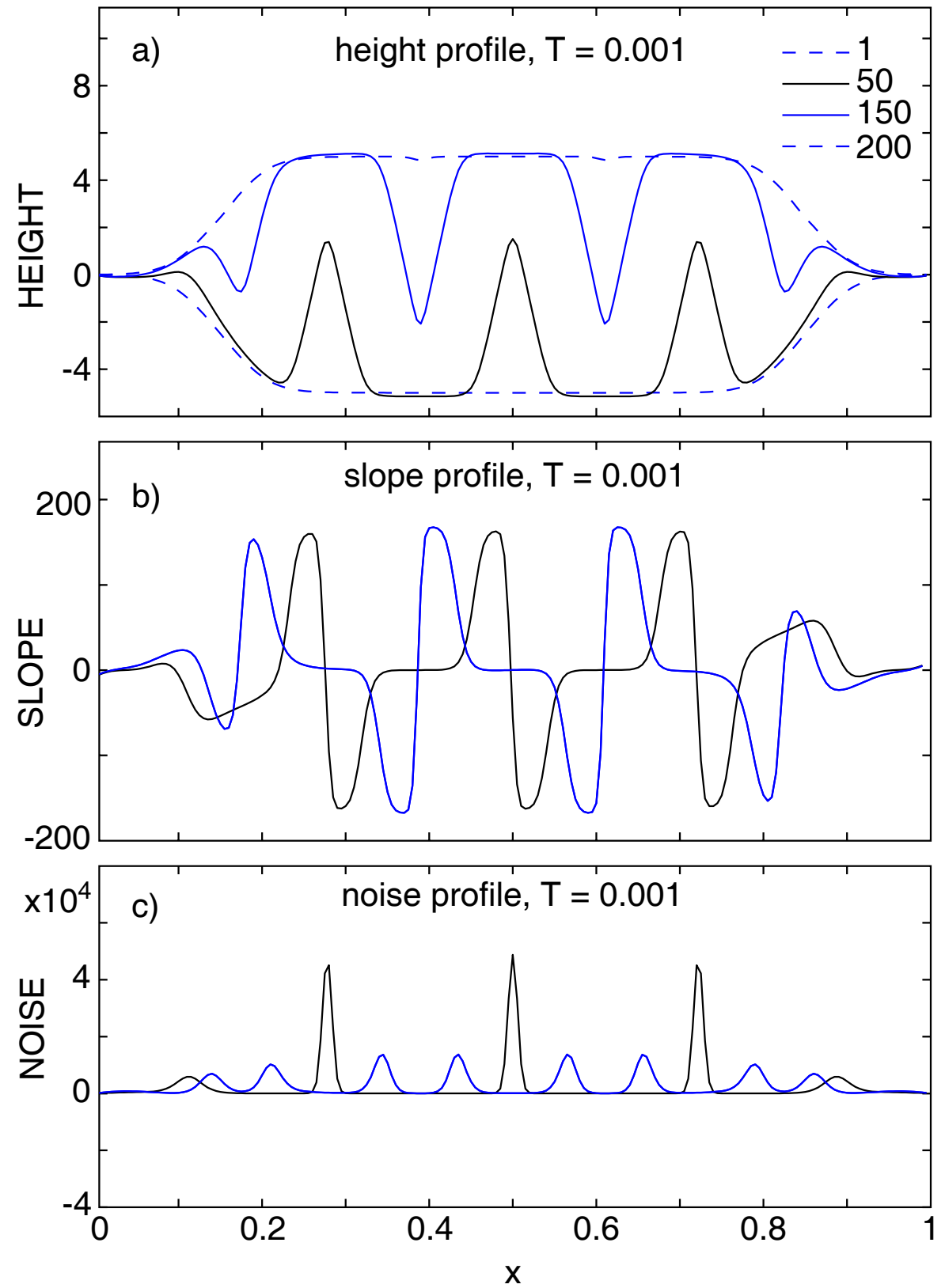

FIG. 10: We depict the transition scenario for transition time $T=0.001$. In a) we show the propagation of the multiple steps or facets in $h$, in b) the associated domain wall pairs in $u$, and in c) the corresponding noise field associated with the nucleation and subsequent propagation of domain walls (arbitrary units). 


\section{Action profile $(T=0.1)$}

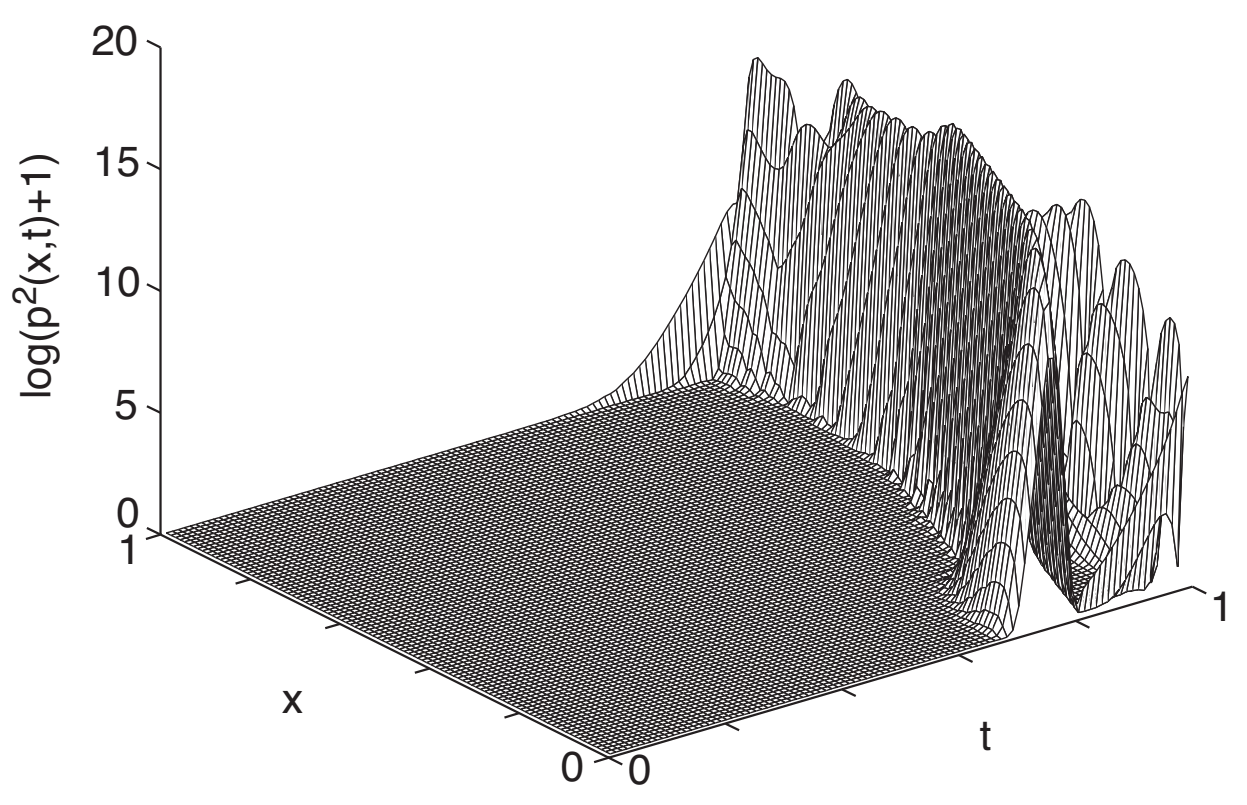

FIG. 11: We plot the squared noise field $p(x, t)^{2}$ or action density as a function of $x$ and $t$ in the case $T=0.1$. The plot show the waiting time aspects of the transition scenario (arbitrary units). 


\section{Action profile ( $\mathrm{T}=0.03)$}

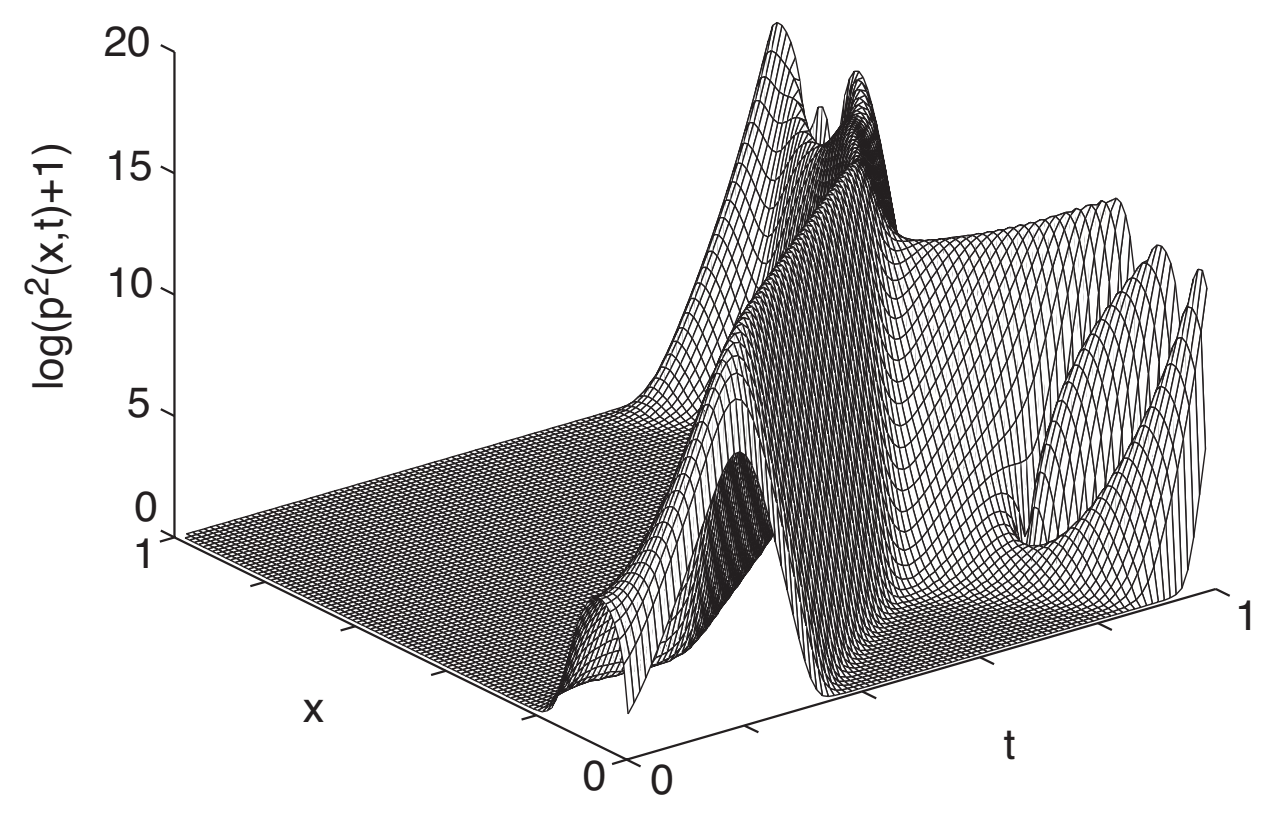

FIG. 12: We plot the squared noise field $p(x, t)^{2}$ or action density as a function of $x$ and $t$ in the case $T=0.03$ (arbitrary units). 


\section{Action profile $(\mathrm{T}=0.01)$}

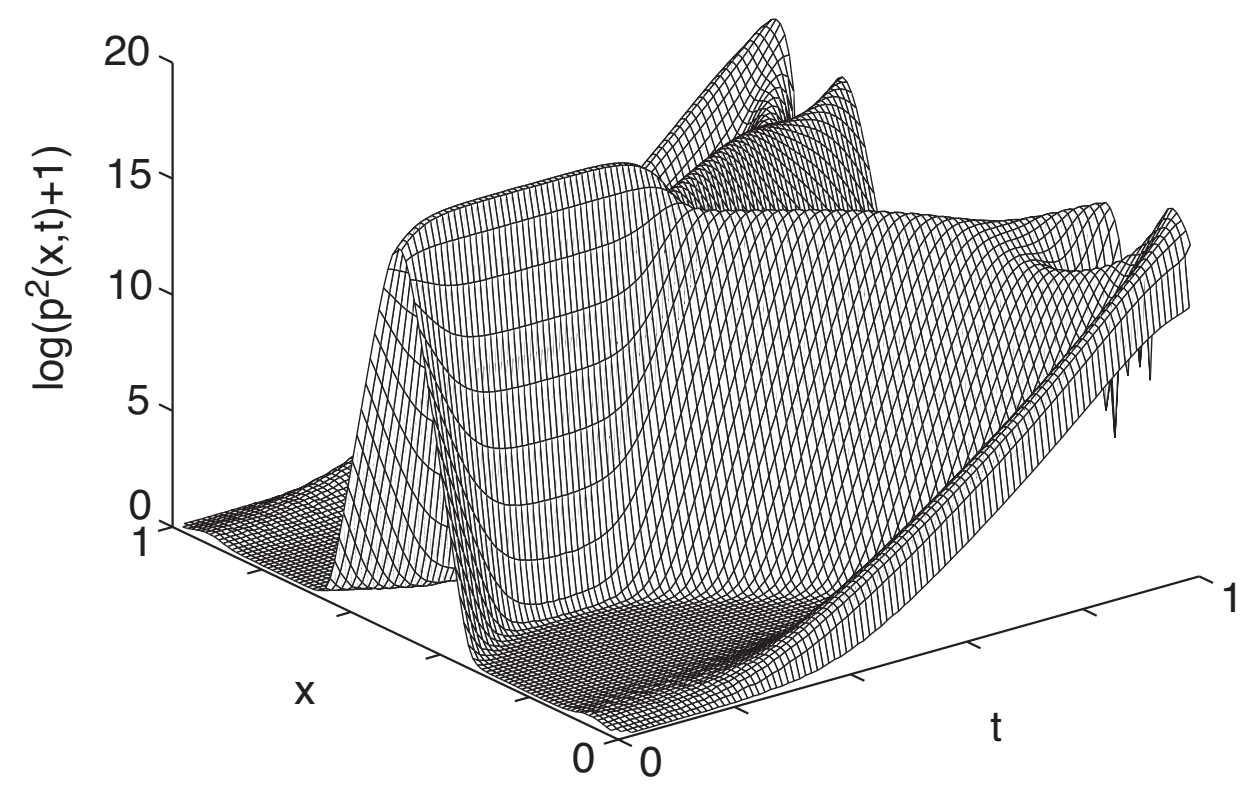

FIG. 13: We plot the squared noise field $p(x, t)^{2}$ or action density as a function of $x$ and $t$ in the case $T=0.01$ (arbitrary units). 


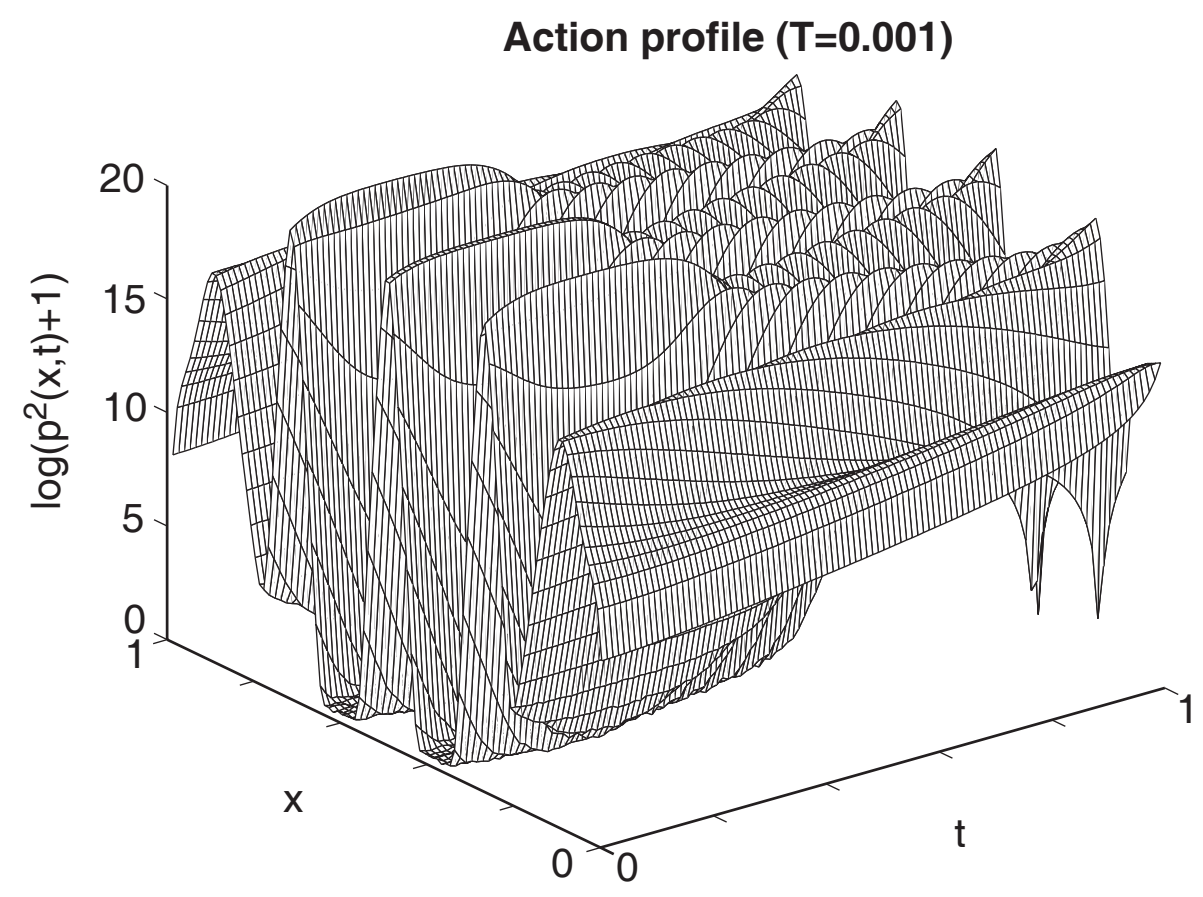

FIG. 14: We plot the squared noise field $p(x, t)^{2}$ or action density as a function of $x$ and $t$ in the case $T=0.001$ (arbitrary units). 


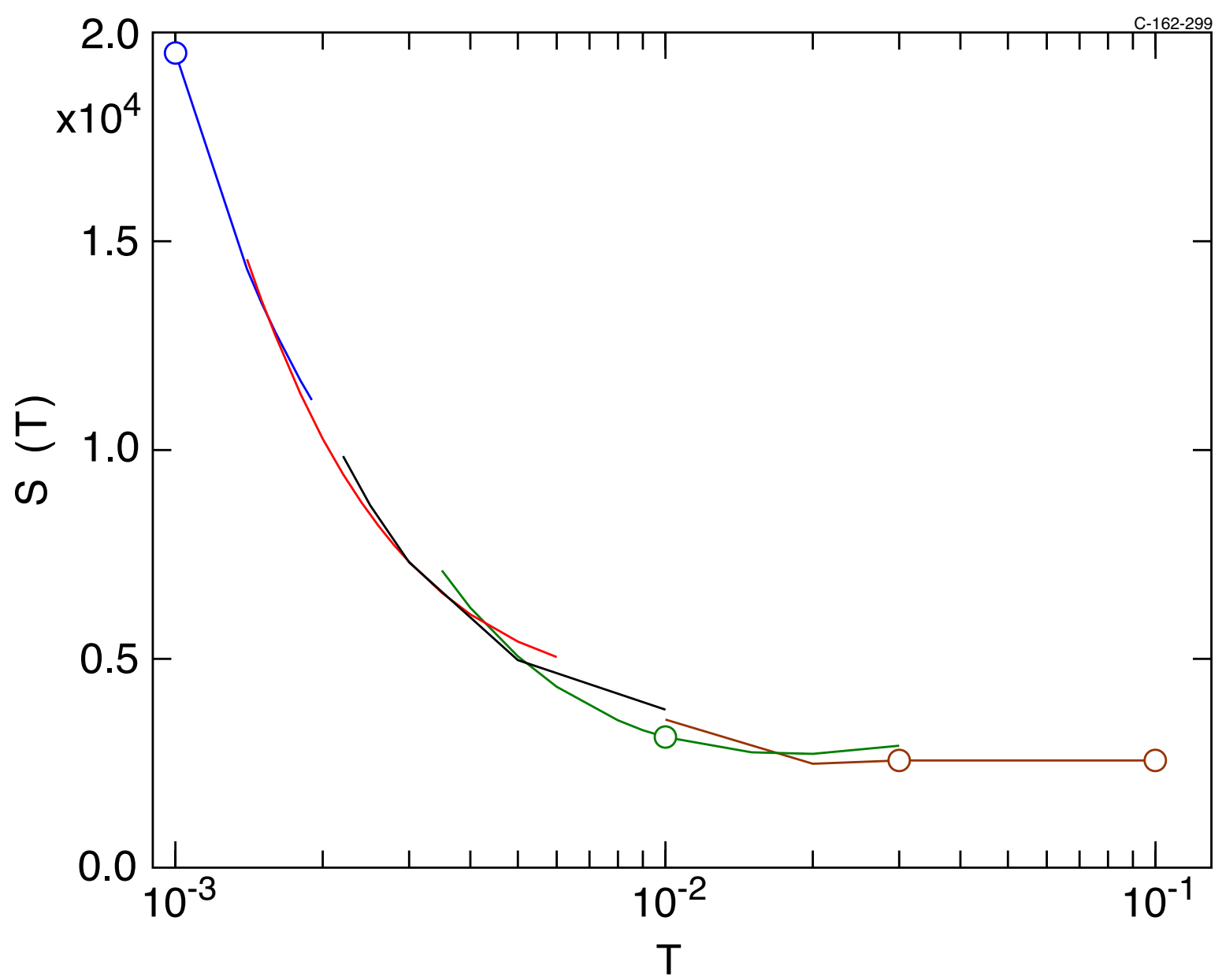

FIG. 15: We depict the action $S(T)$ as a function of the transition time $T$ for five transition scenarios. The circles correspond to the transition pathways for $T=0.01,0.03,0.01,0.001$; the remaining pathways involve one nucleation at the center and one nucleation from the boundary. The plot shows that more domain wall pairs, yielding a lower action, are nucleated at shorter transition times. 


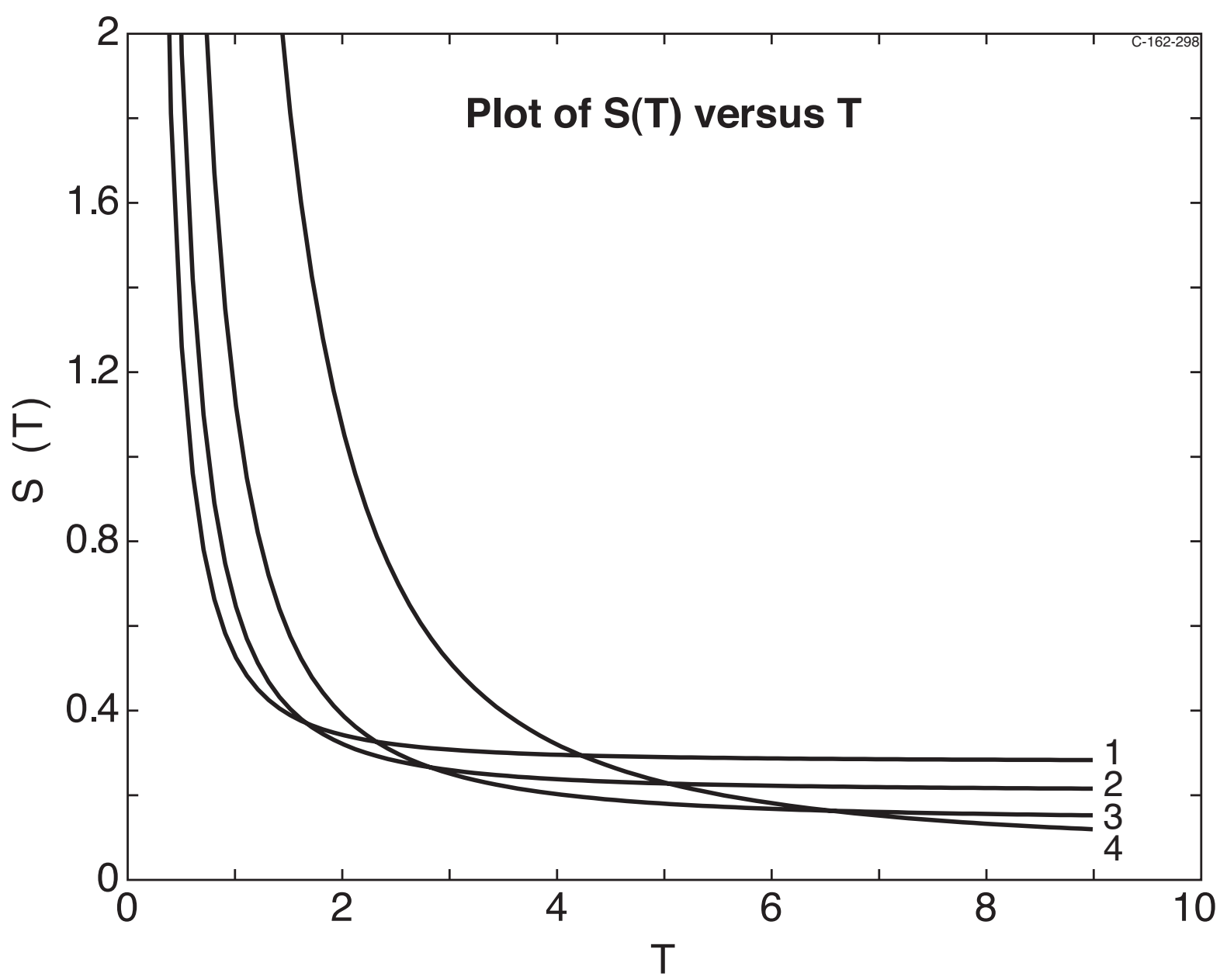

FIG. 16: The action $S(T)$ given by Eq. is plotted as a function of $T$ for transition pathways involving up to 4 domain walls pairs in the slope field. The labeling indicates the number of domain wall pairs. The lowest action and thus the most probable transition is associated with an increasing number of domain wall pairs at shorter transition times (arbitrary units). 


\section{D Height profile $(T=0.02)$}
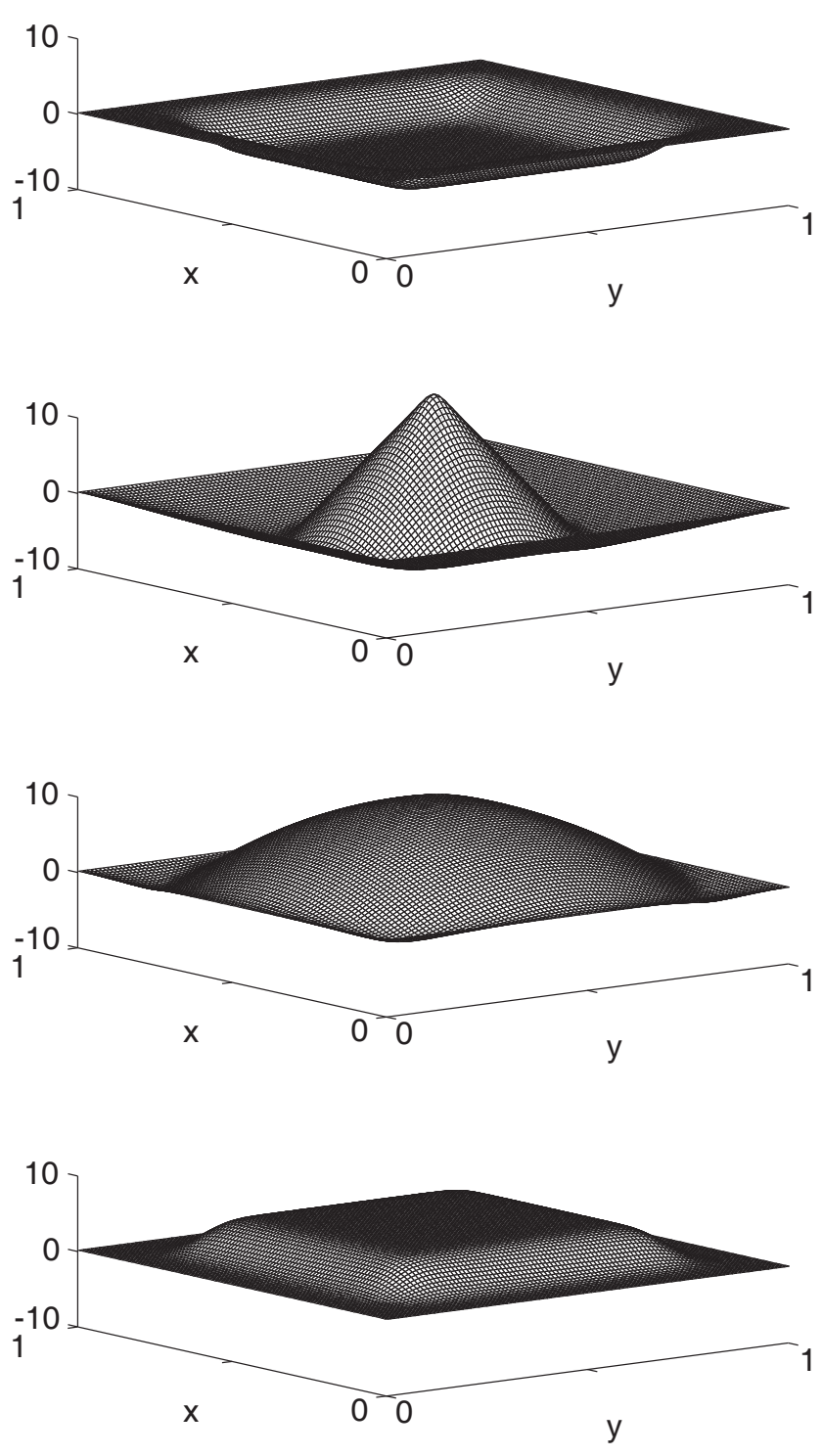

FIG. 17: We depict a 2D long time transition scenario for the height profile from an initial plateau at $h=-5$ to a final plateau at $h=+5$. The transition time is $T=0.02$. The transition takes place subject to the nucleation of a single peak in $h$ at the center. The peak eventually broadens as we approach the final configuration (arbitrary units). 
2D Height profile $(\mathrm{T}=\mathbf{0 . 0 0 2})$
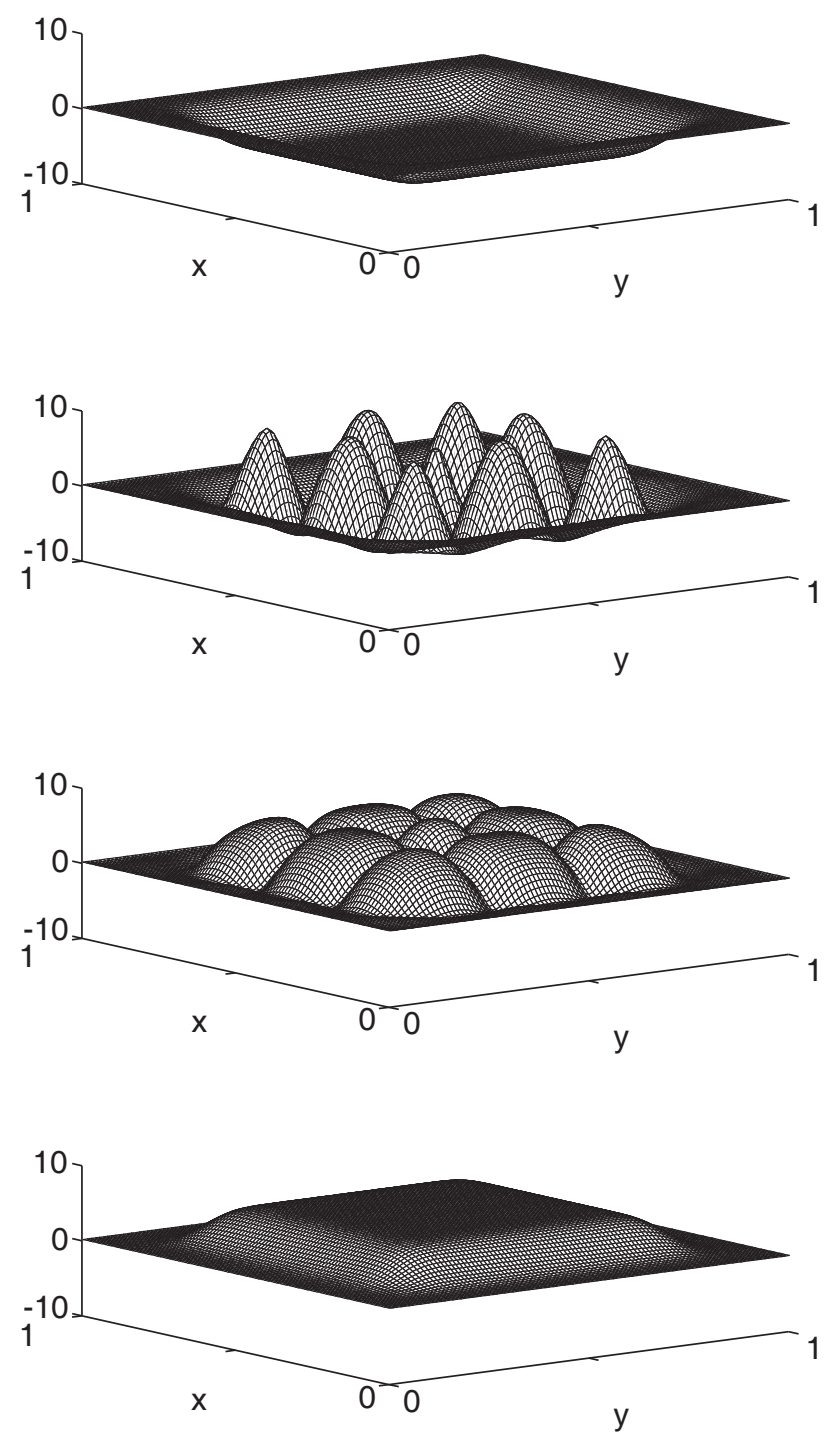

FIG. 18: We depict a 2D short time transition scenario for the height profile from an initial plateau at $h=-5$ to a final plateau at $h=+5$. The transition time is $T=0.002$. The transition takes place subject to a regular pattern of 9 nucleation zones. The peaks eventually broaden as we approach the final configuration (arbitrary units). 